\title{
Zintl ions within framework channels: the complex structure and low-temperature transport properties of $\mathrm{Na}_{4} \mathrm{Ge}_{13}$
}

\author{
Stevce Stefanoski ${ }^{1},{ }^{2}$ Gregory J. Finkelstein ${ }^{3}$, Matthew D. Ward ${ }^{1}$, Tao Zeng ${ }^{4}$, Kaya Wei $^{5}$, \\ Emma S. Bullock ${ }^{1}$, Christine M. Beavers ${ }^{6}$, Hanyu Liu ${ }^{1}$, George S. Nolas ${ }^{5}$, Timothy A. Strobel ${ }^{1 *}$ \\ ${ }^{1}$ Geophysical Laboratory, Carnegie Institution of Washington, Washington, DC 20015, USA \\ ${ }^{2}$ Department of Physics, Benedictine University, Lisle, IL 60532, USA \\ ${ }^{3}$ Seismological Laboratory, California Institute of Technology, Pasadena, CA 91125, USA \\ ${ }^{4}$ Department of Chemistry, Carleton University, Ottawa, ON K1S5B6, Canada \\ ${ }^{5}$ Department of Physics, University of South Florida, Tampa, FL 33620, USA \\ ${ }^{6}$ Advanced Light Source, Lawrence Berkeley National Laboratory, Berkeley, CA 94720, USA
}

Keywords: Zintl phase, thermal conductivity, Raman spectroscopy, germanium, single-crystal diffraction

\begin{abstract}
Single crystals of a complex Zintl compound with the composition $\mathrm{Na}_{4} \mathrm{Ge}_{13}$ were synthesized for the first time using a high-pressure/high-temperature approach. Single-crystal diffraction of synchrotron radiation revealed a hexagonal crystal structure with $\mathrm{P} 6 / m$ space group symmetry that is comprised of a three-dimensional $s p^{3}$ Ge framework punctuated by small and large channels along the crystallographic $c$-axis. $\mathrm{Na}$ atoms are inside hexagonal prism-based Ge cages along the small channels, while the larger channels are occupied by layers of disordered six-fold $\mathrm{Na}$ rings, which are in turn filled by disordered $\left[\mathrm{Ge}_{4}\right]^{4-}$ tetrahedra. This compound is the same as " $\mathrm{Na}_{1-x} \mathrm{Ge}_{3+z}$ " reported previously, but the availability of single crystals allowed for more complete structural determination with a formula unit best described as $\mathrm{Na}_{4} \mathrm{Ge}_{12}\left(\mathrm{Ge}_{4}\right)_{0.25}$. The compound is the first known example of a guest-host structure where discrete Zintl polyanions
\end{abstract}


are confined inside the channels of a three-dimensional covalent framework. These features give rise to a temperature-dependent disorder, as confirmed by first principles calculations and physical properties measurements. The availability of single-crystal specimens allowed for measurement of the intrinsic low-temperature transport properties of this material and revealed its semiconductor behavior, which was corroborated by theoretical calculations.

\section{Introduction}

Zintl-phases are intermetallic compounds that are the product of the reaction between alkali / alkali-earth metals and elements of groups 13-16 [1,2]. Zintl phases are ionic like the classic example of $\mathrm{NaCl}$, and similarly, their component ions achieve an octet via electron transfer from the more electropositive to the more electronegative ion. In contrast, however, the constituent anions in Zintl phases are not necessarily isolated species, and are comprised of multiple atoms bonded together in order to achieve an electron octet. Understanding the science of compounds formed by group 14 elements is fundamental in the search for technologically important materials including photovoltaics, optoelectronics and thermoelectrics. Two important and related chemical systems that form Zintl compounds are those of Si and Ge. There are a variety of these materials with a range of compositions, some of which may have critical applications in energy conversion. Zintl phases in the Si and Ge systems can be generally classified into two families: polyanionic species and framework species [1-4]. Polyanionic species include cluster anions such as $\left[\mathrm{Ge}_{4}\right]^{4-}$, as in the case of $\mathrm{Na}_{4} \mathrm{Ge}_{4}$ and $\mathrm{Na}_{12} \mathrm{Ge}_{17}$, or $\left[\mathrm{Ge}_{9}\right]^{4-}$ such as in $\mathrm{Na}_{12} \mathrm{Ge}_{17}$ [1-4]. Framework species include compositions such as the clathrate-II $\mathrm{Na}_{x} \mathrm{Ge}_{136}(0 \leq x \leq 24)[1$, 5]. Layered Zintl compounds of Si and Ge are also known, as well as Ge and SiGe alloy sheet polymers [6]. However, to the best of our knowledge no three-dimensional compounds that incorporate elements of both framework and polyanion types are known to date.

Recently a new compound in the Na-Ge system, $\mathrm{Na}_{1-x} \mathrm{Ge}_{3+z}$ ( $x$ and $z$ reflect variable $\mathrm{Na}$ and Ge occupancies), was synthesized by thermal decomposition of the Zintl-precursor $\mathrm{Na}_{4} \mathrm{Ge}_{4}$ under vacuum [7-9]. This material was initially synthesized as a microcrystalline powder that also 
contained traces of amorphous Ge. Its structure, as determined by powder diffraction methods, has characteristics qualitatively analogous to aluminosilicate zeolites [9, 10] and Ge clathrates [5]. An unconventional covalent $\mathrm{Ge}$ framework accommodates $\mathrm{Na}$ atoms in two distinct channels giving rise to relatively high disorder, having implications on its physical properties.

Using powder X-ray and neutron diffraction methods, four crystallographically distinct Ge sites with full occupancy were previously determined for the three-dimensional $s p^{3}$ Ge framework, which contains 5-, 6-, and larger 24-membered Ge rings in the $a-b$ plane that create small and large zeolite-like channels along the $c$-axis. Smaller six-membered rings form hexagonal prismbased cages that host $\mathrm{Na}$ atoms with full occupancy. The large 24-member Ge rings form six "lobes" that are concave with respect to the channel center (like a flower with six petals) and each lobe contains one sodium atom (fully or partially occupied depending on the preparation conditions), forming six-fold $\mathrm{Na}$ rings that are layered every $\sim 4 \AA$ along the $c$-axis. The centers of the larger channels were suggested to contain two edge-aligned, hexagonal Ge rings separated along the $c$-axis by only $\sim 1 \AA$. These hexagonal Ge ring pairs occur between each six-fold $\mathrm{Na}$ layer with the Ge ring vertices aligned towards the six $\mathrm{Na}$ atoms in the $a-b$ plane. Unphysical GeGe distances of both in-ring and between ring pairs were rationalized by partial occupancies, although all Ge atoms comprising these rings are described by a single crystallographically unique site and no physical explanation to account for this type of chemical environment is available. It is therefore likely that the crystallographic description and chemical understanding of $\mathrm{Na}_{1-x} \mathrm{Ge}_{3+z}$ is incomplete. Based solely on Rietveld refinements of powder neutron and X-ray diffraction (XRD) data with partially-occupied $\mathrm{Na}$ and Ge sites, the compound was found to have a composition ranging from $\mathrm{NaGe}_{3.24}$ to $\mathrm{NaGe}_{4.34}$, and thus given the general formula $\mathrm{Na}_{1-x} \mathrm{Ge}_{3+z}$, where the "base" structure $\left(\mathrm{NaGe}_{3}\right)$ contains no $\mathrm{Ge}$ in the large channels, but is fully loaded with $\mathrm{Na}$. Within this model, the ideal composition of the full-occupancy structure would be $\mathrm{NaGe}_{3.125}$. In addition, specimens with $65 \%$ of the theoretical density were obtained by careful hot-pressing to avoid decomposition, thus no intrinsic transport properties characterization exists for this material [7]. 
High-pressure/high-temperature (HPHT) methodologies have emerged as promising synthetic routes for preparing new stable and metastable compositions that are otherwise inaccessible by more conventional solid-state synthesis or crystal growth methods [11-14]. The synthesis of single-crystal specimens is a prerequisite to gaining a better understanding of the intrinsic structural and physical properties of materials for a range of technologically important applications. Only a handful of binary Na-Ge compositions are known to date [15-20], and HPHT synthesis has not been explored so far for any of them.

In this paper we report the HPHT synthesis of the " $\mathrm{Na}_{1-x} \mathrm{Ge}_{3+z}$ " phase in the form of high-quality single crystals. We propose an alternate structural model consisting of chains of disordered $\left[\mathrm{Ge}_{4}\right]^{4-}$ Zintl ions in the large channels, which results in a bulk composition of $\mathrm{Na}_{4} \mathrm{Ge}_{13}$. Specimens in the form of agglomerations of single crystals allowed for measurement of the intrinsic low-temperature transport properties, including electrical and thermal conductivity and the Seebeck coefficient, for the first time.

\section{Experimental Methods}

Synthesis

$\mathrm{Na}_{4} \mathrm{Ge}_{13}$ was synthesized from a $\mathrm{Na}: \mathrm{Ge}=1: 3$ mixture that was ground using a ceramic mortar and pestle for one hour inside a high-purity Ar glovebox and loaded into a Ta capsule (Caution: $\mathrm{Na}$ and Na-Ge mixtures are highly reactive in air / moisture and should only be handled in an inert atmosphere). The capsule was then introduced into an 18/11 multianvil assembly using a graphite heater with $\mathrm{ZrO}_{2}$ insulation. A W-Re C-type thermocouple, imbedded in an $\mathrm{Al}_{2} \mathrm{O}_{3}$ plug, was used for accurate temperature control. The mixture was pressurized in a 1500 ton multianvil press at a rate of $10 \mathrm{bar} / \mathrm{h}$ (oil pressure) to a specimen pressure of $4 \mathrm{GPa}$, and reacted at $800{ }^{\circ} \mathrm{C}$ in two steps: preheating at $400{ }^{\circ} \mathrm{C}$ for 30 minutes, to avoid a blowout of the molten $\mathrm{Na}$, followed by a reaction at the final temperature for one hour, after which the sample was quenched by switching off the power. The recovered sample was easily removed from the Ta capsule and then washed with distilled water to remove the unreacted $\mathrm{Na}$. The stability of the crystals in water and air was verified by measuring identical XRD patterns over time (one year). 


\section{$X$-ray diffraction}

Initial powder X-ray diffraction (PXRD) analyses were performed on crushed specimens using a Bruker D8 diffractometer with $\mathrm{Cu} \mathrm{K}_{\alpha}$ radiation and Vantec 500 area detector. Single-crystal diffraction measurements were performed at the Advanced Photon Source, sector 16 (HPCAT) on beamline 16-BMD. Monochromatic X-rays $(0.30998 \AA)$ with a beam size of $\left(5 \times 5 \mu \mathrm{m}^{2}\right.$, FWHM) were focused on the sample and room-temperature diffraction was collected using a MAR345 image plate operating in 2300 mode. Oscillation scans were collected on one axis between omega $=-30$ to $+25^{\circ}$ and angular step scans were conducted every 1 degree. Peak positions and intensities were extracted and unit cell parameters were refined in GSE_ADA and RSV software packages, respectively [21]. Single crystal diffraction experiments were also conducted on Beamline 11.3.1 at the Advanced Light Source of Lawrence Berkeley National Laboratory. A blocky silver crystal with dimensions of $\sim 0.05 \times 0.05 \times 0.02 \mathrm{~mm}^{3}$ was mounted on a MiTeGen ${ }^{\circledR}$ sample mount, then placed in the 100(2) K nitrogen cold stream provided by an Oxford Cryosystems Cryostream 700 Plus low-temperature apparatus. An approximately full sphere of data to $2 \theta_{\max }=40^{\circ}$ was collected using a Bruker D85 diffractometer equipped with a Photon 100 CMOS detector. Synchrotron X-rays were monochromated to a wavelength of $0.7749 \AA$ using silicon (111). The data were integrated using the program SAINT v8.27b. A multi-scan correction for absorption was applied with the program SADABS v2014/5. A total of 46264 reflections were collected, of which 1410 were unique $[\mathrm{R}(\mathrm{int})=0.0323]$, and 1371 were observed $[\mathrm{I}>2 \sigma(\mathrm{I})]$.

Structure solutions were achieved using intrinsic phasing (SHELXT) and refinements were completed by full-matrix least-squares minimization of $\mathrm{F}^{2}$ using the SHELX-14 suite of programs within the PLATON software package [22]. 47 parameters were refined with one restraint [23, 24]. Space group assignment was based upon systematic absences, $E$-statistics, agreement factors for equivalent reflections and successful refinement of the structure. Atomic positions were standardized using the program STRUCTURE TIDY $[25,26]$. Crystallographic images were made using the program CRYSTALMAKER (CrystalMaker Software Ltd., 
CrystalMaker®.) [27]. Further crystallographic details are given in Tables 1-2 and the supporting information (SI).

\section{Electron microprobe analysis}

Single-crystal specimens were mounted in epoxy and polished to a surface roughness $<1 \mu \mathrm{m}$. The samples were analyzed using a JEOL 8530F field emission electron probe, operating at 10 $\mathrm{kV}$ and $10 \mathrm{nA}$. The Na-K $\alpha$ and Ge-L $\alpha$ lines were measured in order to minimize peak overlap. The standards used were pure Ge metal and DJ35, a Na-bearing glass, and the detection limits were 70 ppm and 200 ppm for $\mathrm{Na}$ and Ge, respectively. A total of 27 analyses were collected on multiple crystals within a single analytical session.

\section{Raman Spectroscopy}

Raman spectra from samples were obtained in the back-scatter geometry using a $532 \mathrm{~nm}$ laser $(\sim 1 \mathrm{~mW}$ power) as the excitation source. Laser light was focused using a $20 \times$ Mitutoyo objective lens (0.40 NA) and scattered light was passed through a $50 \mu \mathrm{m}$ spatial filter and low-frequency holographic notch filters. Raman light was dispersed off of an 1800 groove/mm grating and focused onto a liquid-nitrogen-cooled, charge-coupled device detector.

\section{Low-Temperature Transport Properties}

The steady-state thermal conductivity, $\kappa$, Seebeck coefficient, $S$, and four-probe resistivity, $\rho$, measurements on single-crystal specimens of approximate dimensions $0.1 \mathrm{~mm} \times 0.1 \mathrm{~mm} \times 0.1$ $\mathrm{mm}$ were conducted in a custom radiation-shielded vacuum probe in the temperature range between 12 and $300 \mathrm{~K}[28,29]$. Conservative estimates of the room temperature maximum uncertainties in the measurements of $\kappa, S$ and $\rho$ are $30 \%, 6 \%$, and $30 \%$, respectively. The large uncertainties estimated for the $\kappa$ and $\rho$ measurements are due to the relatively large contacts as compared to the size of the specimens.

\section{Calculations}


Density functional theory calculations were performed using the Vienna ab initio simulation package (VASP-5.3.5) [30-33] with the PBE functional [34, 35], the projector augmented-wave (PAW) potentials [36, 37], and a $270 \mathrm{eV}$ plane-wave energy cutoff. The Monkhorst-Pack scheme [38] was used to generate the K points. The density of K points was systematically increased until the convergence of $10^{-5} \mathrm{eV} /$ atom in calculated enthalpy was reached. The LOBSTER-1.2.0 program $[39,40]$ was used to calculate the projected density of states. The GAMESS-US program package [41, 42] was used for the molecular calculation, with the same functional and the cc-pVTZ basis set [43].

Raman spectra were calculated using density functional perturbation theory (DFPT) using a Python code [44] in combination with VASP. In order to calculate off-resonance Raman activity of a mode, the derivative of the polarizability (or macroscopic dielectric tensor) with respect to that normal mode coordinate is needed. To obtain the Raman intensity for each mode, phonons at the $\Gamma$-point were calculated, followed by calculation of the macroscopic dielectric tensor [45].

\section{Results and Discussion}

\section{Synthesis and initial characterization}

Single crystals of $\mathrm{Na}_{4} \mathrm{Ge}_{13}$ were synthesized for the first time using the HPHT approach discussed in the previous section. The resulting samples consisted of polycrystalline agglomerates of visibly reflective crystals with grain sizes ranging between $\sim 50-500 \mu \mathrm{m}$. The crystals were found to be brittle upon mechanical agitation, but remained chemically stable in open air (for a period of $>1$ year). Several crystals were crushed into coarse powder and analyzed using PXRD. The resulting PXRD patterns were found to qualitatively agree with the JCPDS cards for the previously reported hexagonal $\mathrm{Na}_{1-x} \mathrm{Ge}_{3+z}$ structure with $a \approx 15.08 \AA$ and $c \approx 3.98$ $\AA$, although relative peak intensities were not accurately determined due to limited grain statistics. The slightly expanded lattice parameters at $298 \mathrm{~K}(+0.1 \%$ in $a$ and $+0.2 \%$ in $b)$ could be related to the increased or more complete $\mathrm{Na} / \mathrm{Ge}$ filling as compared with samples prepared at low pressure. We note that complete or increased guest filling was also observed in $\mathrm{Na}-\mathrm{Si}$ clathrate samples produced at high pressure $[13,46]$. 


\section{Composition from WDS and EDS}

In order to measure the composition of this phase, Wavelength-Dispersive Spectroscopy (WDS) and Energy Dispersive Spectroscopy (EDS) analyses were performed using a JEOL 8530F electron probe at the Carnegie Institution of Washington (CIW) on multiple single-crystal specimens from two independent synthesis runs. The WDS results reveal a uniform composition across the 27 independent measurements, an indication of a pure phase of $\mathrm{Na}$ and $\mathrm{Ge}$. The only other elements detected by EDS were carbon and oxygen, which originate from the carbon coating for electrical conductivity and the native oxide layer, respectively. The measured Na:Ge atomic ratio of 1:3.25 \pm 0.05 indicates a nominal structural formula of $\mathrm{Na}_{4} \mathrm{Ge}_{13}$, which is consistent with the previous observations from PXRD analysis of $\mathrm{Na}_{1-x} \mathrm{Ge}_{3+z}$. In terms of the previous model, we note, however, that 1:3.25 would require a significant number of $\mathrm{Na}$ vacancies and it is anticipated that vacancies would decrease at high pressure $[13,46]$.

\section{Single-crystal structure}

The crystal structure of $\mathrm{Na}_{4} \mathrm{Ge}_{13}$ is hexagonal and was solved in the space group $P 6 / m$ (No. 175), which is the same space group obtained previously for $\mathrm{Na}_{1-x} \mathrm{Ge}_{3+z}$ [7]. The structural model was refined using synchrotron diffraction collected at $100 \mathrm{~K}$. Details of the crystallographic structure and refinement are given in Tables 1-2 and the SI.

Refinement and solution of the framework structure for $\mathrm{Na}_{4} \mathrm{Ge}_{13}$ was straightforward, but the refinement of the Ge atoms in the channels was complicated by partial occupancies (Table 2). Refinement of the occupancies of the Ge1 and Ge6 sites gives partial occupancies of 0.116(1) and $0.242(4)$, resulting in $1.87 \mathrm{Ge} 1 / \mathrm{Ge} 6$ atoms per unit cell and an overall composition of $\mathrm{Na}_{4} \mathrm{Ge}_{12.935}$. As the elemental composition was determined to be $\mathrm{Na}_{4} \mathrm{Ge}_{13}$ from independent WDS measurements, and in order to achieve charge balance, a constraint equation of $2=12 f_{1}+2 f_{2}$, where $f_{1}$ and $f_{2}$ are the occupancies of Gel and Ge6, was used. Refinement using this constraint equation gives occupancies of $0.125(1)$ and $0.250(6)$ for $\mathrm{Ge} 1$ and Ge6, respectively. Additional disorder is displayed by the $\mathrm{Na}$ atoms within the larger channel. Initial refinements resulted in 
one $\mathrm{Na}$ site on a $6 j$ position. The anisotropic displacement parameters were significantly elongated and indicated that the site should be split. This splitting results in two Na1 sites, Na1a and $\mathrm{Na} 1 \mathrm{~b}$, on $6 j$ and $12 l$ positions, with partial occupancies of $1 / 2$ and $1 / 4$, respectively. This structure results in a total composition of $\mathrm{Na}_{8} \mathrm{Ge}_{26}$ per unit cell. The disorder of the Nal sites might be further explained by modulation of the channel along $c$, but precession images of the data collection yielded no evidence of such modulation. We note that it is possible to refine the data down to an R-factor of 0.0175 by removing the constraint on Ge1/Ge6 and by allowing the $\mathrm{Na1a} / \mathrm{b}$ occupancy to fluctuate. This approach results in Ge1/Ge6 occupations that do not have reasonable chemical interpretations and gives an overall chemical compositions that is far outside of the independent WDS measurements.

Table 1. Crystallographic Details for $\mathrm{Na}_{4} \mathrm{Ge}_{13}$

$$
\begin{gathered}
\text { Crystal System } \\
\text { Space Group } \\
a, b / \AA \\
c / \AA \\
V / \AA^{3}
\end{gathered}
$$

Formula unit composition

$$
\text { Z }
$$

Density, $\rho_{\text {calc }}\left(\mathrm{g} / \mathrm{cm}^{3}\right)$

$T / \mathrm{K}$

Wavelength $(\lambda / \AA)$

Absorption Coefficient $\left(\mathrm{mm}^{-1}\right)$

Reflections Collected

Data/Parameters/Restraints

$R_{\text {int }}$

$R(\mathrm{~F})[\mathrm{I}>2 \sigma(\mathrm{I})]^{\mathrm{a}}$

$R_{w}\left(\mathrm{~F}_{\mathrm{o}}^{2}\right)^{\mathrm{b}}$
Hexagonal

$P 6 / m$

$15.0275(5)$

3.9602(2)

$774.50(6)$

$\mathrm{Na}_{4} \mathrm{Ge}_{12}\left(\mathrm{Ge}_{4}\right)_{0.25}$

2

4.441

100(2)

0.7749

31.32

46264

1410/48/1

0.0323

0.0204

0.0502 
${ }^{\mathrm{a}} R(F)=\sum\left\|F_{\mathrm{o}}|-| F_{\mathrm{c}} \quad\right\| / \sum\left|F_{\mathrm{o}} \quad\right|$ for $F_{\mathrm{o}}{ }^{2}>2 \sigma\left(F_{\mathrm{o}}{ }^{2}\right) .{ }^{b} R_{\mathrm{w}}\left(F_{\mathrm{o}}{ }^{2}\right)=\left\{\sum\left[w\left(F_{\mathrm{o}}^{2}-F_{\mathrm{c}}{ }^{2}\right)^{2}\right] / \sum w F_{\mathrm{o}}{ }^{4}\right\}^{1 / 2}$ for all data. $w^{-1}=\sigma^{2}\left(F_{o}^{2}\right)+\left(0.0181 F_{o}^{2}\right)^{2}$ for $F_{o}^{2} \geq 0 ; w^{-1}=\sigma^{2}\left(F_{o}^{2}\right)$ for $F_{o}^{2}<0$.

Table 2: Atomic positions, Atomic Displacement Parameters $\left(U_{e q}\right.$ or $\left.U_{i s o}\right)$, and Site Occupancy Factors $(S O F)$ for $\mathrm{Na}_{4} \mathrm{Ge}_{13}$.

\begin{tabular}{ccccccc}
\hline Atom & $\begin{array}{r}\text { Wyckoff } \\
\text { position }\end{array}$ & $\boldsymbol{x}$ & $\boldsymbol{y}$ & $\boldsymbol{Z}$ & $\boldsymbol{U}_{\text {eq }}$ or $\boldsymbol{U}_{\text {iso }}$ & SOF \\
\hline $\mathrm{Ge} 1$ & $12 l$ & $0.0271(2)$ & $0.1088(2)$ & $0.4074(9)$ & $0.0302(8)$ & $0.125(1)$ \\
$\mathrm{Ge} 2$ & $6 k$ & $0.0417(1)$ & $0.4473(1)$ & $1 / 2$ & $0.0048(1)$ & 1 \\
$\mathrm{Ge} 3$ & $6 k$ & $0.3695(1)$ & $0.1033(1)$ & $1 / 2$ & $0.0063(1)$ & 1 \\
$\mathrm{Ge} 4$ & $6 j$ & $0.1524(1)$ & $0.5186(1)$ & 0 & $0.0048(1)$ & 1 \\
$\mathrm{Ge} 5$ & $6 j$ & $0.4831(1)$ & $0.1841(1)$ & 0 & $0.0053(1)$ & 1 \\
$\mathrm{Ge} 6$ & $2 e$ & 0 & 0 & $0.0757(10)$ & $0.0298(9)$ & $1-6 \cdot \mathrm{Ge} 1$ \\
$\mathrm{Na} 1 \mathrm{a}$ & $6 j$ & $0.0696(3)$ & $0.2739(3)$ & 0 & $0.0237(6)$ & $1 / 2$ \\
$\mathrm{Na} 1 \mathrm{~b}$ & $12 l$ & $0.0636(4)$ & $0.2427(4)$ & $0.0626(15)$ & $0.0237(6)$ & $1 / 4$ \\
$\mathrm{Na} 2$ & $2 d$ & $1 / 3$ & $2 / 3$ & $1 / 2$ & $0.0102(4)$ & 1 \\
\hline
\end{tabular}

The title compound is the same as the previously reported $\mathrm{Na}_{1-x} \mathrm{Ge}_{3+z}$, but is more accurately described as $\mathrm{Na}_{4} \mathrm{Ge}_{13}$; the unit cell contains two formula units of $\mathrm{Na}_{4} \mathrm{Ge}_{12}\left(\mathrm{Ge}_{4}\right)_{0.25}$ in the hexagonal space group $C_{6 h}^{1}-P 6 / m$ (No. 175). The structure is composed three of crystallographically independent $\mathrm{Na}$ sites and six Ge sites. All sites have symmetry $m$.., except for atoms Ge1, Ge6, Na1b and Na2, which have site symmetries of 1, 6.., 1 and $\overline{6}$.., respectively.

The structure of $\mathrm{Na}_{4} \mathrm{Ge}_{13}$ can be described as a three-dimensional framework of Ge atoms with two types of channels, one being occupied by only $\mathrm{Na}$ and the other by both $\mathrm{Na}$ and $\mathrm{Ge}_{4}$ tetrahedra, along the $c$-axis (Figure 1). The Ge framework is composed of four Ge sites, three of which $(\mathrm{Ge} 2, \mathrm{Ge} 4$, and $\mathrm{Ge} 5)$ are four-coordinate to other Ge atoms in distorted tetrahedral 
environment and one (Ge3) that is three-coordinate to other $\mathrm{Ge}$ atoms in a pyramidal environment, and is analogous to the Ge framework in $\mathrm{Na}_{1-x} \mathrm{Ge}_{3+z}$. The four-coordinate Ge atoms have $\mathrm{Ge}-\mathrm{Ge}$ distances ranging from 2.460(1) $\AA$ to 2.537(1) $\AA$ and the three-coordinate Ge atoms have distances of 2.494(1) $\AA$ to 2.497(1) $\AA$ (Table S1 in the SI). These distances may be compared to those seen in $\mathrm{Ba}_{6} \mathrm{Ge}_{25}$, which also has a three-dimensional $\mathrm{Ge}$ framework-like structure, and has three-coordinate $\mathrm{Ge}-\mathrm{Ge}$ distances ranging from 2.498(1) $\AA$ to 2.552(2) $\AA$ and four-coordinate $\mathrm{Ge}-\mathrm{Ge}$ distances ranging from 2.475(2) $\AA$ to 2.581(2) $\AA$ [47]. From this coordination and bonding environment, it is expected that the $\mathrm{Ge}$ atoms in the framework of $\mathrm{Na}_{4} \mathrm{Ge}_{13}$ will have formal charges comparable to those seen in $\mathrm{Ba}_{6} \mathrm{Ge}_{25}$, with three- and fourcoordinate Ge atoms having formal charges of -1 and 0 , respectively. This gives a $\mathrm{Ge}$ framework of $\left[(3 b) \mathrm{Ge}^{-}\right]_{3}\left[(4 b) \mathrm{Ge}^{0}\right]_{9}$ with a formal charge of -3 .

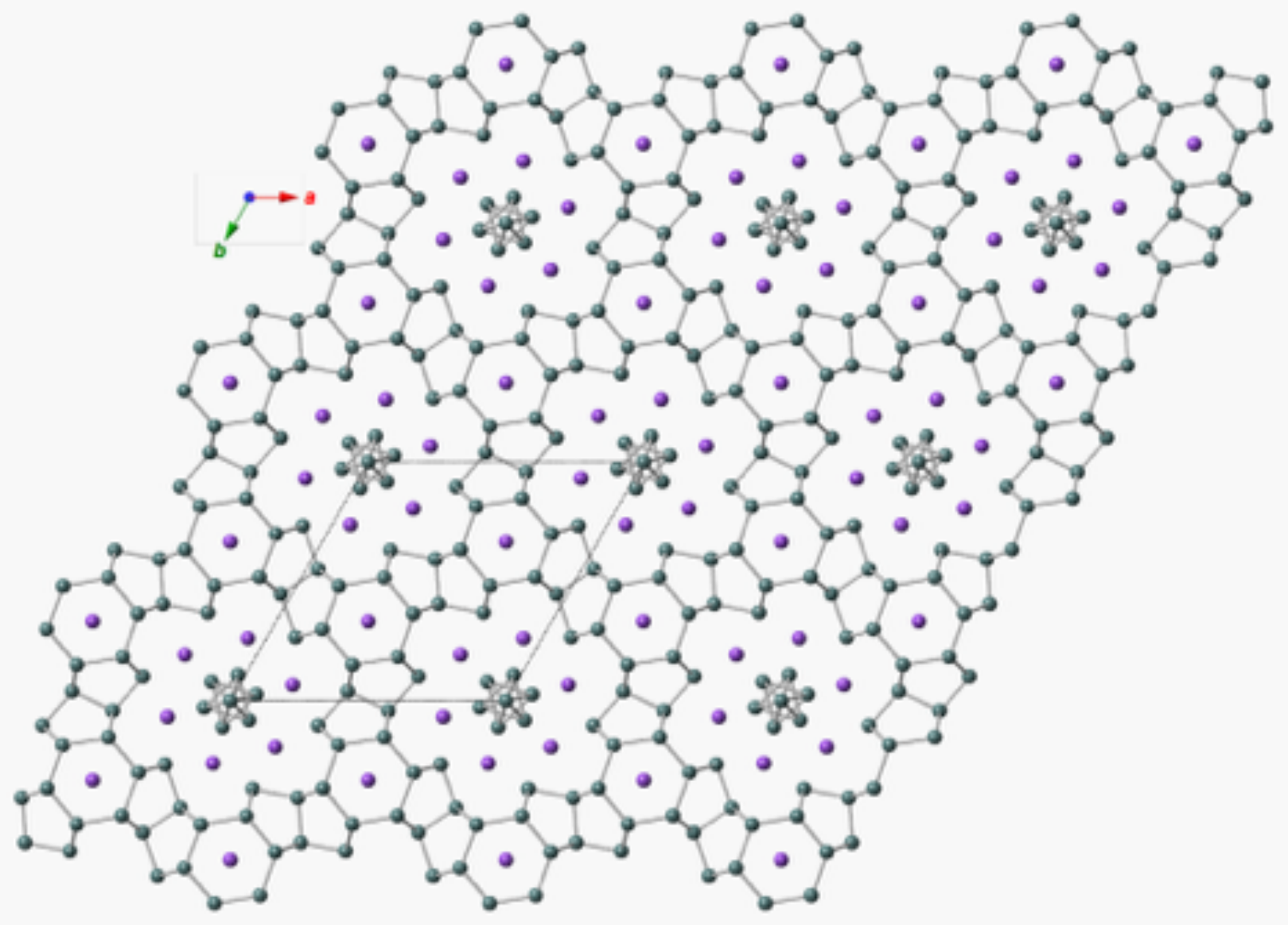


Figure 1. Three-dimensional framework structure of $\mathrm{Na}_{4} \mathrm{Ge}_{13}$ with $\mathrm{Na}^{+}$ions and $\left[\mathrm{Ge}_{4}\right]^{4-}$ tetrahedra within the smaller and larger channels, respectively. Purple and green- colored spheres represent $\mathrm{Na}$ and $\mathrm{Ge}$ atoms, respectively. The $\mathrm{Na}$ l atom is shown as a single site in the large channel for clarity.

The framework structure contains 5-, 6-, and 24-member Ge rings that create two types of zeolite-like channels along the $c$-axis. The smaller of these channels is occupied by $\mathrm{Na} 2$ atoms which are twelve-coordinate and located between two six-membered rings composed of Ge4 and Ge5 atoms. These Na2-Ge4/5 distances range from 3.196(1) $\AA$ to 3.221(1) $\AA$. The larger channel is formed by rings of $24 \mathrm{Ge}$ atoms, six of which are three-coordinate Ge3 atoms that are expected to carry a formal charge of -1 (Figure 2a). This large channel is more complex with two Ge sites, Ge1 and Ge6, at the center of the channel. These central Ge atoms are surrounded by $\mathrm{Na} 1 \mathrm{a}$ and $\mathrm{Na} 1 \mathrm{~b}$ atoms at the edges of the channel that each sit between two Ge3 atoms of the 24-membered ring. When not accounting for partial occupancies, the Ge1 atoms in the center of the channel form two edge-aligned, six-membered rings analogous to those seen in the $\mathrm{Na}_{1}$ ${ }_{x} \mathrm{Ge}_{3+z}$ model with unreasonable inter- and intra-ring Ge-Ge distances of 0.733(7) $\AA$ to 1.647(4) $\AA$ and 1.475(2) $\AA$. In the $\mathrm{Na}_{1-x} \mathrm{Ge}_{3+z}$ model, partial occupancy was used to explain these unreasonable distances, but no chemical explanation was suggested. Two Ge6 atoms sit between two sets of Ge1 six-membered ring dimers. Without partial occupancies, unreasonable 0.602(8) $\AA ̊$ Ge6-Ge6 distances are observed. The closest Ge6-Ge1 distance is 1.974(4) $\AA$. The Ge6 site, which is absent in the refinements for the $\mathrm{Na}_{1-\mathrm{x}} \mathrm{Ge}_{3+\mathrm{z}}$ model, is crucial to creating a reasonable chemical description that achieves charge balance. Refinement of the occupancies for Ge1 and Ge6 was described above and gives respective values of 0.125(1) and 0.250(6). As Ge1 sits on a 12-fold site and Ge6 sits on 2-fold site, these partial occupancies give an approximate ratio of 3:1 for Ge1:Ge6. This results in $1.5 \mathrm{Ge} 1$ and $0.5 \mathrm{Ge} 6$ atoms per unit cell and $0.75 \mathrm{Ge} 1$ and $0.25 \mathrm{Ge} 6$ atoms per formula unit.

As described above, the Ge framework will have an overall charge of -3 and each of the four $\mathrm{Na}$ atoms is expected to have a formal charge of +1 . This leaves a net charge of +1 to be balanced 
by the one Ge1/Ge6 atom per formula unit. In order to achieve an average formal charge of -1 for $\mathrm{Ge} 1$ and $\mathrm{Ge} 6$, it is reasonable to expect some real Ge1-Ge6 bonding interactions. Within the six-membered rings, reasonable Ge1-Ge1 distances of 2.554(4) Å are seen between Ge atoms residing at any of the first, third or fifth (meta) ring positions. To account for the appropriate Ge1:Ge6 composition, further Ge1-Ge6 interactions with distances of 2.417(4) $\AA$ and 2.522(4) $\AA$ allow for the formation of $\left[\mathrm{Ge}_{4}\right]^{4-}$ tetrahedra (Figure $2 \mathrm{~b}$ ). These are comparable with the distances of 2.545(1) $\AA$ to 2.583(1) $\AA$ seen in $\mathrm{A}_{14} \mathrm{ZnGe}_{16}(\mathrm{~A}=\mathrm{K}, \mathrm{Rb})$ [48]. When accounting for the partial occupancies for Ge1 and Ge6, none of the unusually short distances will persist with the proposed model of $\left(\mathrm{Ge}_{4}\right)^{4-}$ tetrahedra. It should also be noted that it is not unreasonable to expect $\left(\mathrm{Ge}_{4}\right)^{4-}$ tetrahedra in the present compound as the original synthesis of $\mathrm{Na}_{1-\mathrm{x}} \mathrm{Ge}_{3+\mathrm{z}}$ proceeded from $\mathrm{Na}_{4} \mathrm{Ge}_{4}$. Thus, $\mathrm{Na}_{4} \mathrm{Ge}_{13}$ may be charge balanced as $\left[\mathrm{Na}^{+}\right]_{4}\left[(3 b) \mathrm{Ge}^{-}\right]_{3}\left[(4 b) \mathrm{Ge}^{0}\right]_{9}\left[\left(\mathrm{Ge}_{4}\right)^{4-}\right]_{0.25}$. Discrete ordering of these $\left[\mathrm{Ge}_{4}\right]^{4-}$ tetrahedra within individual channels would alleviate the need to split the Na1 position, however, it appears that the occupation is likely not ordered, either within or between channels, as no evidence for modulation was observed in the pseudo-precession diffraction images (Figure 2c-d).

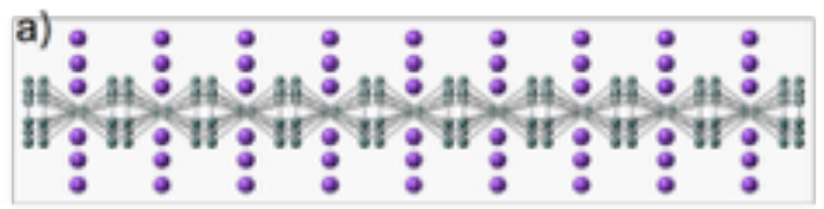

c)
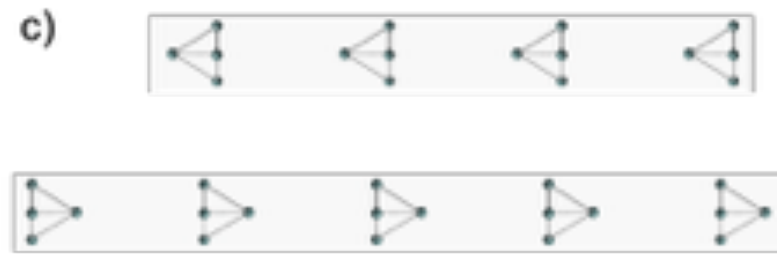

b)

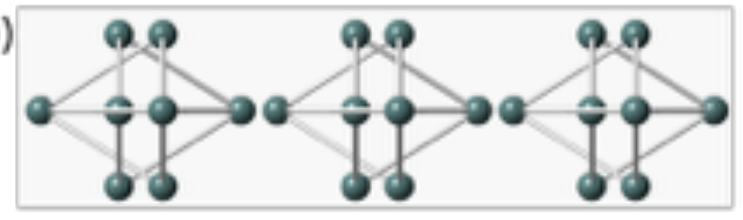

d)

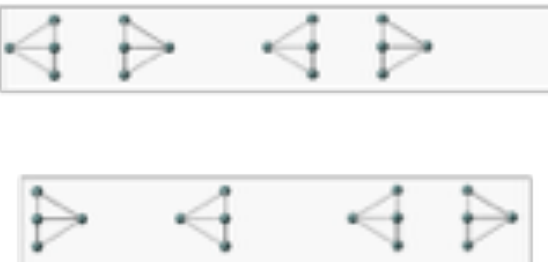

Figure 2. a) Occupation of the large channels along the $c$-axis by $\mathrm{Na}^{+}$and $\left[\mathrm{Ge}_{4}\right]^{4-}$ tetrahedra. $\mathrm{Na} 1$ atoms are shown on a single site for clarity. b) View of the $\left[\mathrm{Ge}_{4}\right]^{4-}$ tetrahedra without partial occupancies. Tetrahedra can adopt eclipsed or staggered conformations by rotating $60^{\circ}$ around 
the $c$-axis. c) Partial occupation of the Ge1 and Ge6 sites with ordering of discrete $\left[\mathrm{Ge}_{4}\right]^{4-}$ tetrahedra within or between channels. d) Partial occupation of Ge1 and Ge6 sites without ordering of discrete $\left[\mathrm{Ge}_{4}\right]^{4-}$ tetrahedra within or between channels.

\section{Raman spectroscopy}

$\mathrm{Na}_{4} \mathrm{Ge}_{13}$ exhibits a complex Raman spectrum, similar to other Ge-based framework structures

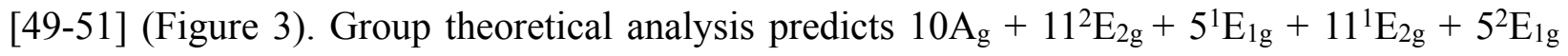
Raman active modes for the Ge framework and $\mathrm{Na}$ atoms within the structure, with an additional 18 Raman active modes possible for the channel $\left[\mathrm{Ge}_{4}\right]^{4-}$ tetrahedra. Given the complex nature of the spectra, we performed density functional perturbation theory (DFPT) calculations to aid in the mode assignments and to better understand the nature of the atomic vibrations.

The Raman spectrum of $\mathrm{Na}_{4} \mathrm{Ge}_{13}$ is almost entirely described by contributions from the covalent Ge framework and encapsulated $\mathrm{Na}$ atoms. This is not unexpected given the ratio of framework atoms to tetrahedra within the unit cell. The most intense mode at $130 \mathrm{~cm}^{-1}$ is dominated by symmetric framework bending motion. Broad contributions at higher frequencies of $184 \mathrm{~cm}^{-1}$ and $223 \mathrm{~cm}^{-1}$ represent modes associated with in-phase and out-of-phase ring breathing, while the highest frequency modes grouped near $245 \mathrm{~cm}^{-1}$ and $268 \mathrm{~cm}^{-1}$ are dominated by in-plane and out-of-plane Ge-Ge stretching. Contributions from the $\left[\mathrm{Ge}_{4}\right]^{4-}$ tetrahedra occur at similar frequencies to other $\mathrm{M}_{4} \mathrm{Ge}_{4}$ Zintl compounds [52-54], but exhibit strong overlap with the framework modes. A sharp contribution at $270 \mathrm{~cm}^{-1}$ could be a signature of the Zintl ion's symmetric stretch, but it is difficult to isolate the contributions from the tetrahedra experimentally, given the dominance of the framework modes. 


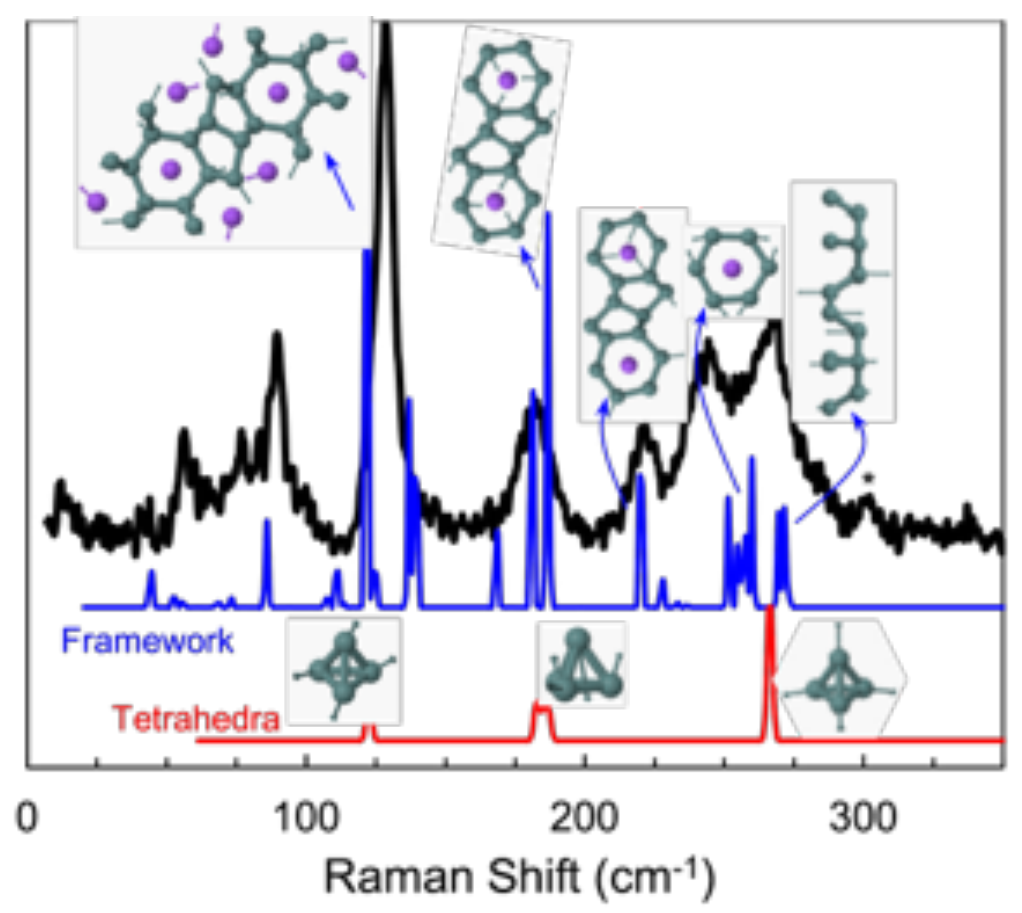

Figure 3. Experimental Raman spectrum (black) of $\mathrm{Na}_{4} \mathrm{Ge}_{13}$ compared with DFPT calculations. Calculated spectra are separated into contributions from the Ge framework with Na atoms (blue) and $\left[\mathrm{Ge}_{4}\right]^{4-}$ tetrahedra (red). A scaling factor of 1.06 was applied to the frequencies of the calculated spectra, which are shown as Gaussian profiles with an arbitrary width. Graphical representations of the character of prominent modes are shown, where arrows indicate the eigenvectors of the atomic displacements. The asterisk indicates a minor contribution from $d$-Ge impurity.

\section{Stability, chemical bonding and electronic structure}

Density Functional Theory (DFT) calculations were performed in order to investigate the electronic structure of $\mathrm{Na}_{4} \mathrm{Ge}_{13}$. As mentioned above, every unit cell of the crystal contains only half of a $\left[\mathrm{Ge}_{4}\right]^{4-}$ tetrahedron. A supercell with twice the lattice parameter along the $c$-axis was therefore used to account for the tetrahedral disorder, labeled as “c $\times 2$ " in Figure 4. 


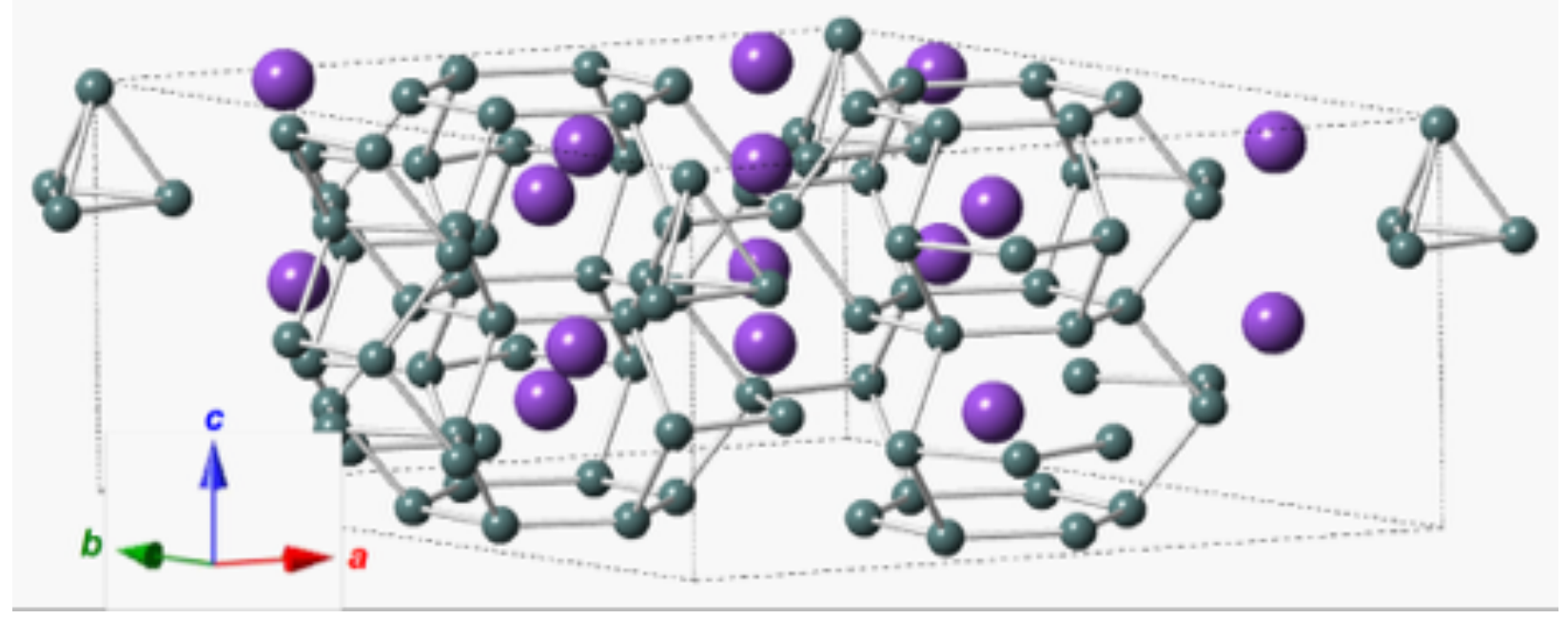

Figure 4. Unit cell of the $\mathrm{c} \times 2$ structure $\left(\mathrm{Na}_{16} \mathrm{Ge}_{52}\right)$. $\mathrm{Na}$ and $\mathrm{Ge}$ atoms are represented by purple and green spheres, respectively.

The model unit cell is constructed following the crystal structure previously discussed using only a single site $(6 j)$ for $\mathrm{Na} 1$. The number of the respective atoms is doubled along with the $c$-axis. 36 of the $52 \mathrm{Ge}$ atoms are 4-coordinated (4c). The leftover 16 are 3-coordinated (3c). A purely ionic Zintl-Klemm picture will have each of the 16 3c Ge atoms take an electron from the $\mathrm{Na}$ atoms to form a lone pair to satisfy the octet structure. The optimized unit cell has space group symmetry $P 3$ (No. 143). The lattice parameters are $15.27 \AA$ and $8.08 \AA$ for $(a, b)$ and $c$, respectively. They are slightly larger than the $15.03 \AA$ and $3.96 \times 2 \AA$ in Table 1 . The bond lengths are also slightly larger. For instance, within the tetrahedral $\left[\mathrm{Ge}_{4}\right]^{4-}$, the Ge-Ge distance between the tip and the trigonal base atoms is $2.66 \AA$, larger than the $2.52 \AA$ experimental value (the cif file is provided in the SI). The Ge-Ge distances in the trigonal base are $2.60 \AA$, longer than the $2.55 \AA$ of the experimental counterpart. The calculated Ge-Ge distances are close to the value of $2.67 \AA$ for the $T_{d}\left[\mathrm{Ge}_{4}\right]^{4-}$ obtained from molecular calculations (see SI).

Taking the body centered cubic (BCC) $\mathrm{Na}$ and diamond-like $\mathrm{Ge}(d-\mathrm{Ge})$ structures at $1 \mathrm{~atm}$ pressure as references, the enthalpy of formation for 


$$
16 \mathrm{BCC}-\mathrm{Na}+52 d-\mathrm{Ge}=\mathrm{Na}_{16} \mathrm{Ge}_{52}
$$

is exothermic by $0.31 \mathrm{eV} / \mathrm{Na}$ atom. Compared to the Zintl-precursor $\mathrm{Na}_{4} \mathrm{Ge}_{4}$ [1], the formation of the $\mathrm{Na}_{16} \mathrm{Ge}_{52}$ structure with the following reaction formula

$$
4 \mathrm{Na}_{4} \mathrm{Ge}_{4}+36 d-\mathrm{Ge}=\mathrm{Na}_{16} \mathrm{Ge}_{52}
$$

is exothermic by $0.16 \mathrm{eV} / \mathrm{Na}$ atom. The $\mathrm{Na}_{4} \mathrm{Ge}_{4}$ structure has $\sim 4 \AA$ adjacent Ge-Ge distances, which negates any covalent interaction. The Ge-Ge covalent bonding in $\mathrm{Na}_{16} \mathrm{Ge}_{52}$ is responsible for the energy lowering and it seems likely that $\mathrm{Na}_{4} \mathrm{Ge}_{13}$ is a thermodynamically stable compound on the convex hull. The experimental observation of its formation from both decomposition of $\mathrm{Na}_{4} \mathrm{Ge}_{4}$ and directly from the elements supports this postulation, but the presence of another, as-of-yet undiscovered germanium-rich binary with lower formation enthalpy could change this understanding.

The electronic density of states (DOS) for the $\mathrm{c} \times 2$ structure is shown in Figure 5(a). There is a small gap of $0.16 \mathrm{eV}$, an indication of the semiconductor nature of $\mathrm{Na}_{16} \mathrm{Ge}_{52}$. The direct band gap is at the $\mathrm{K}$ point in the first Brillouin zone (see Figure S5 in SI for the band structure). The projected DOS (pDOS) on the different types of atoms are shown in Figure 5(b). The figure is obtained from calculations using the LOBSTER package. Different binning and smearing used in the calculation results in band gap closure. This does not impact our conclusion on the semiconductor nature of the material since the pDOS delivers a qualitative picture only. The two types of $3 \mathrm{c}$ Ge atoms are differentiated as those forming the tetrahedra (th) and those at the sides (side) of the large channel containing the 6-membered Na-rings. The two types of $\mathrm{Na}$ are also differentiated based on their Wyckoff positions in the original (before taking twice the $c$-length) unit cell with the $P 6 / m$ space group symmetry. 

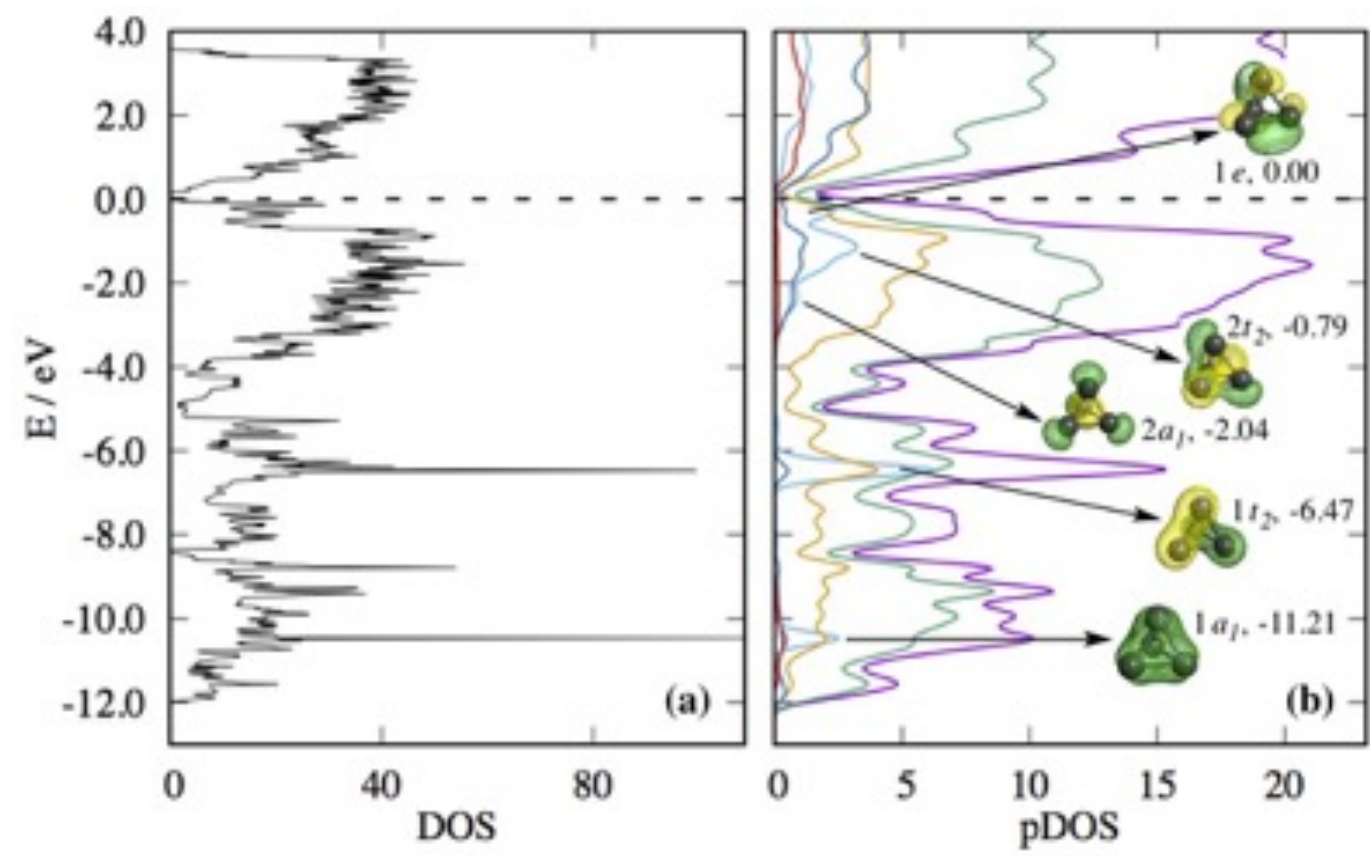

total -

$\mathrm{Ge} 4 \mathrm{c}-$

$\mathrm{Ge} 3 \mathrm{c}$ th

$\mathrm{Ge} 3 \mathrm{c}$ side

$\mathrm{Na} 6 \mathrm{j}$

$\mathrm{Na} 2 \mathrm{~d}$

Figure 5. DOS (a) and the projected DOS (b) of the $\mathrm{c} \times 2 \mathrm{Na}_{16} \mathrm{Ge}_{52}$ structure. The units of the DOS plot are states/eV/unit cell. The DOS and pDOSs have been shifted so that the Fermi energy is at $0 \mathrm{eV}$. The numbers following the $\left[\mathrm{Ge}_{4}\right]^{4-}$ cluster orbital labels are their orbital energies in $\mathrm{eV}$ obtained through molecular DFT calculation. The $1 e$ energy has been taken as the zero reference.

The pDOS of the $3 c$ (th) Ge atoms correspond well with the valence molecular orbitals of $\left[\mathrm{Ge}_{4}\right]^{4-}$, as indicated by the arrows in Figure 5(b) (see Figure S1 in SI for larger figures of the orbitals). To be consistent with the Fermi energy setting, we have chosen the energy of the highest occupied molecular orbitals, $1 e$, as zero. $\left[\mathrm{Ge}_{4}\right]^{4-}$ is isoelectronic and isostructural to the classic $\mathrm{P}_{4}$ molecule. Their valence orbitals are hence in one-to-one correspondence. The cyan pDOS peaks of $3 c$ (th) Ge at $-10.5 \mathrm{eV}$ and $-6.5 \mathrm{eV}$ in Figure 5 correspond to the $1 a_{1}$ and $1 t_{2}$ orbitals. The hump ranging from -3 to $0 \mathrm{eV}$ consists of three peaks, corresponding to the $2 a_{1}, 2 t_{2}$, and $1 e$ orbitals. The area under each of the five peaks correlate well with the degeneracy of the corresponding orbital, e.g., the $1 a_{1}$ peak area is 0.92 state/unit cell, while the $1 t_{2}$ peak area is 2.67 states/unit cell, about three times larger. 


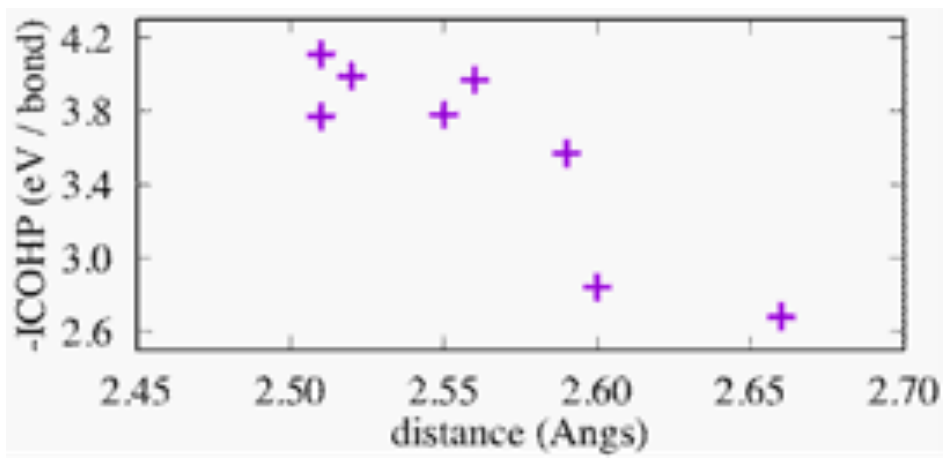

Figure 6. - ICOHP vs. Ge-Ge distance. The dashed line is added to guide comparison.

We also calculated the integrated crystal orbital Hamilton populations up to the Fermi level [55] for symmetrically unique Ge-Ge pairs using the LOBSTER package. For the atoms Ge2-5, only the symmetrically unique pairs in the $\mathrm{P} 6 / \mathrm{m}$ space group are considered. The results are presented in Table S2 and Figure 6. Please note that the more negative the ICOHP value (more positive ICOHP), the stronger bonding interaction will be. In Figure 6, we clearly see the general trend that a longer distance corresponds to a weaker bonding interaction. It is interesting to compare the -ICOHP of Ge1-Ge6 (2.66 $\AA, 2.68 \mathrm{eV} /$ bond) with those of the Ge pairs with similar distances in $\mathrm{BaGe}_{3}$ calculated by Zurek and Yao [56]. The three pairs with $2.68 \AA$ distances in Table 2 of Ref. [56] have $\sim 1.8 \mathrm{eV} /$ bond-ICOHPs. The bonding interactions are weaker than the Ge1-Ge6 here. The difference in bonding interaction reflects the difference in stability between the $\left[\mathrm{Ge}_{3}\right]^{2-}$ moieties in $\mathrm{BaGe}_{3}$ and the $\left[\mathrm{Ge}_{4}\right]^{4-}$ clusters in $\mathrm{Na}_{4} \mathrm{Ge}_{13}$.

The atomic charges are evaluated using Bader's Quantum Theory of Atoms in Molecules (QTAIM) scheme $[57,58]$. The $3 \mathrm{c}(\mathrm{th})$ Ge charges are evaluated to be -1.0 for the three atoms in the triangular base and -0.8 for the one at the top, consistent with the $\left[\mathrm{Ge}_{4}\right]^{4-}$ description of the tetrahedron. The charges of the twelve $3 c$ (side) Ge atoms are all around -0.4 . Their -1 formal charges are shared by the $4 \mathrm{c}$ Ge atoms, especially the twenty four that form the 6-membered rings perpendicular to the $c$-axis in the small channels. Half of the twenty four are calculated to possess -0.4 effective charge, while the other half -0.3 . They are the closest to the $\mathrm{Na}$ atoms (by $\sim 3.2 \AA$ ) in the small channels and are hence the easiest to accept the electrons lost by the $\mathrm{Na}$ atoms. The leftover $4 \mathrm{c}$ Ge atoms possess -0.1 effective charge. The effective charges of all $\mathrm{Na}$ 
atoms are 0.0. The ionic Zintl picture is pronounced. The $3 c($ th) Ge atoms in the trigonal base are almost coplanar as the six $3 c$ (side) Ge atoms. They provide electrostatic stabilization for the $\mathrm{Na}$ ions above and below (Figure 7).

The crystal structure of $\mathrm{Na}_{4} \mathrm{Ge}_{13}$ shows a large degree of static disorder for the $\left[\mathrm{Ge}_{4}\right]^{4-}$ tetrahedra, which can adopt a number of configurations that are related by simple rotations. To investigate the possibility of flipping these tetrahedra within the large channels, calculations for a $\mathrm{Na}_{32} \mathrm{Ge}_{104}$ unit cell were conducted wherein the lattice parameter along the $c$-axis was quadrupled (called $\mathrm{c} \times 4$ structure below). This second doubling along the $c$-direction does not lead to any Peierls distortion. The energy of the optimized $c \times 4$ structure is exactly twice of the $c \times 2$. It takes only $0.06 \mathrm{eV} /$ unit cell to flip one of the tetrahedra to have the structure shown in Figure 7. Such a head-to-head arrangement of the tetrahedra is symbolically labeled by " $><$ ", where the tip of " $>$ " denotes the tip of the tetrahedron. The $0.06 \mathrm{eV}$ energy difference corresponds to $10 \%$ Boltzmann population at room temperature. It can be seen that the tetrahedron only needs to rotate by approximately $70^{\circ}$ to have any of its three trigonal Ge atoms point in the opposite direction, i.e., a flipping. The entropy favorability and the slight $0.06 \mathrm{eV} /$ unit cell enthalpy unfavorability suggest that the tetrahedra can tumble and have the tip-to-tip/base-to-base arrangement within one chain along the $c$-axis. The tumbling is not a free rotation. Otherwise, a significant amount of diffuse scattering would be observed in the XRD-it would appear to be chains of $\mathrm{Ge}_{4} \mathrm{spheres}$ Note that the $0.06 \mathrm{eV} /$ unit cell enthalpy cost is for forming the " $><><><$ " type of configuration, which is of higher energy than the " $<<<>>>$ " and " $>>><<<"$ counterparts. The two chainsymbols here just indicate a random flipping, not that every second tetrahedron flips as in Figure 7. The $0.06 \mathrm{eV} /$ unit cell should hence be taken as the maximum enthalpy in flipping once every second tetrahedron. More likely, the $\mathrm{XRD}$ has revealed the $\mathrm{Ge}_{4}$ distribution with random tetrahedral flipping. 


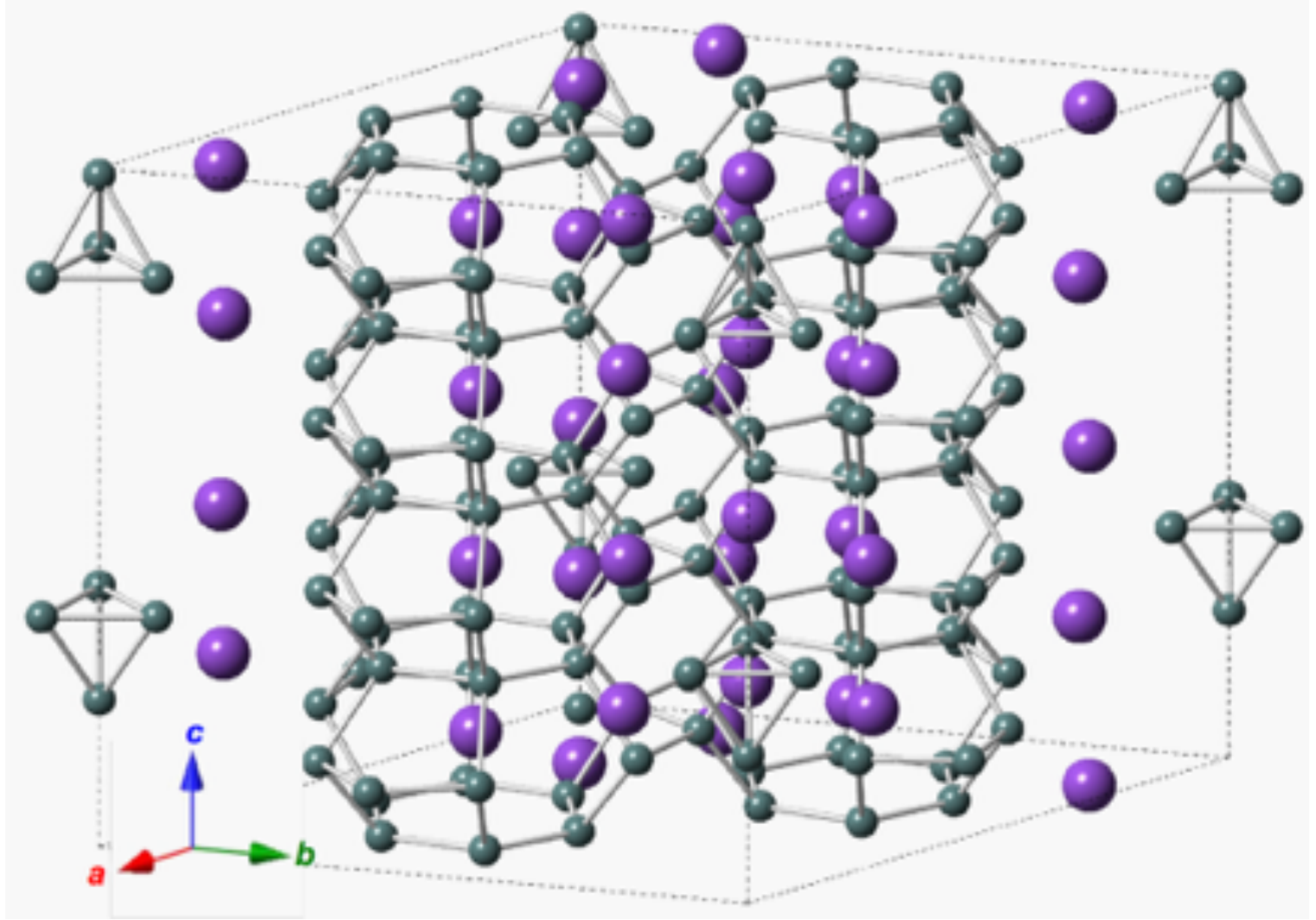

Figure 7. The $\mathrm{c} \times 4$ unit cell with one $\mathrm{Ge}_{4}$ tetrahedron flipped.

For the $\mathrm{c} \times 4$ structure, we have also examined the possibility of rotating the tetrahedron about the $c$-axis, i.e., to change from the eclipsed to staggered configuration. The " $<<<<"$ tetrahedra arrangement is maintained in this case. The optimized supercell structure with the staggered arrangement is only $0.003 \mathrm{eV} /$ unit cell lower in enthalpy, essentially isothermic to the eclipsed configuration. But it requires a significant radial relocation of $\mathrm{Na}$ atoms in the surrounding 6membered Na rings (Figure S4 and the relevant discussion in the SI). This kinetically hinders the formation of the staggered tetrahedra configuration, once the eclipsed configuration is formed, and the other way around.

The synthesis of $\mathrm{Na}_{8} \mathrm{Ge}_{2} 6$ was carried out at a pressure of $4 \mathrm{GPa}$, as a result of which the $\mathrm{c} \times 2$ calculated lattice parameters are reduced to 14.74 and $7.90 \AA$ for $(a, b)$ and $c$. The tip-to-base GeGe distance in the tetrahedron and the one between the two trigonal base Ge atoms are 2.64 and $2.59 \AA$, respectively, only 0.02 and $0.01 \AA$ shorter than in the $1 \mathrm{~atm}$ calculation. This is 
reasonable as there is a significant amount of empty space between the tetrahedron and its neighboring entities; the high pressure acts directly on the bulk Ge cage, not the chains of tetrahedra. The Ge-Ge distances in the bulk cage are more affected by the pressure. For example, the calculated $\mathrm{Ge}-\mathrm{Ge}$ distances in the 6-membered rings along the $c$-axis are reduced from 2.59 $\AA$ and $2.56 \AA$ to $2.52 \AA$ and $2.49 \AA$.

At $4 \mathrm{GPa}$, the formation enthalpy for the $\mathrm{c} \times 2$ structure for the following reaction:

$$
\text { 16BCC-Na }+52 d-\mathrm{Ge}=\mathrm{Na}_{16} \mathrm{Ge}_{52}
$$

is $-0.57 \mathrm{eV} / \mathrm{Na}$ atom, almost twice more exothermic than at $1 \mathrm{~atm}(-0.31 \mathrm{eV} / \mathrm{Na})$. The higher pressure favors the more compact $\mathrm{c} \times 2$ structure as some $\mathrm{Na}$ atoms have been doped in the larger Ge-channels. The $\mathrm{c} \times 4$ calculation under $4 \mathrm{GPa}$ shows that it takes similar enthalpy $(0.055$ vs 0.061 / unit cell) to flip the tetrahedron to have " $><$ " chain. The pressure does not make a difference. The DOS and band structure of the $\mathrm{c} \times 2$ structure at $4 \mathrm{GPa}$ are shown in Figure $\mathrm{S} 5$ in the SI. There is no significant difference from the $1 \mathrm{~atm}$ counterparts (Figure S5). The band gap is $0.18 \mathrm{eV}$, similar to the $0.16 \mathrm{eV}$ at $1 \mathrm{~atm}$. Overall, the pressure does not modify the electronic structure considerably. The projected DOS are similar to those shown in Figure 5.

\section{Transport properties}

The HPHT synthesis methodology described in this paper allowed for the availability of fullydense, homogenous $\mathrm{Na}_{4} \mathrm{Ge}_{13}$ in the form of agglomerations of single crystals. The transport properties of $\mathrm{Na}_{4} \mathrm{Ge}_{13}$ were measured directly on these crystals, employing a previously described methodology [59-61]. Specimens from three separate synthesis runs were independently measured, producing transport properties that are in agreement with each other. As a result of the complex hexagonal structure of this compound, the transport properties are likely anisotropic and therefore the data obtained from the polycrystalline specimens should be interpreted as an average measure of the transport in the different crystallographic directions. Figure 8 shows $S$ and $\kappa$ as a function of temperature for $\mathrm{Na}_{4} \mathrm{Ge}_{13}$. 


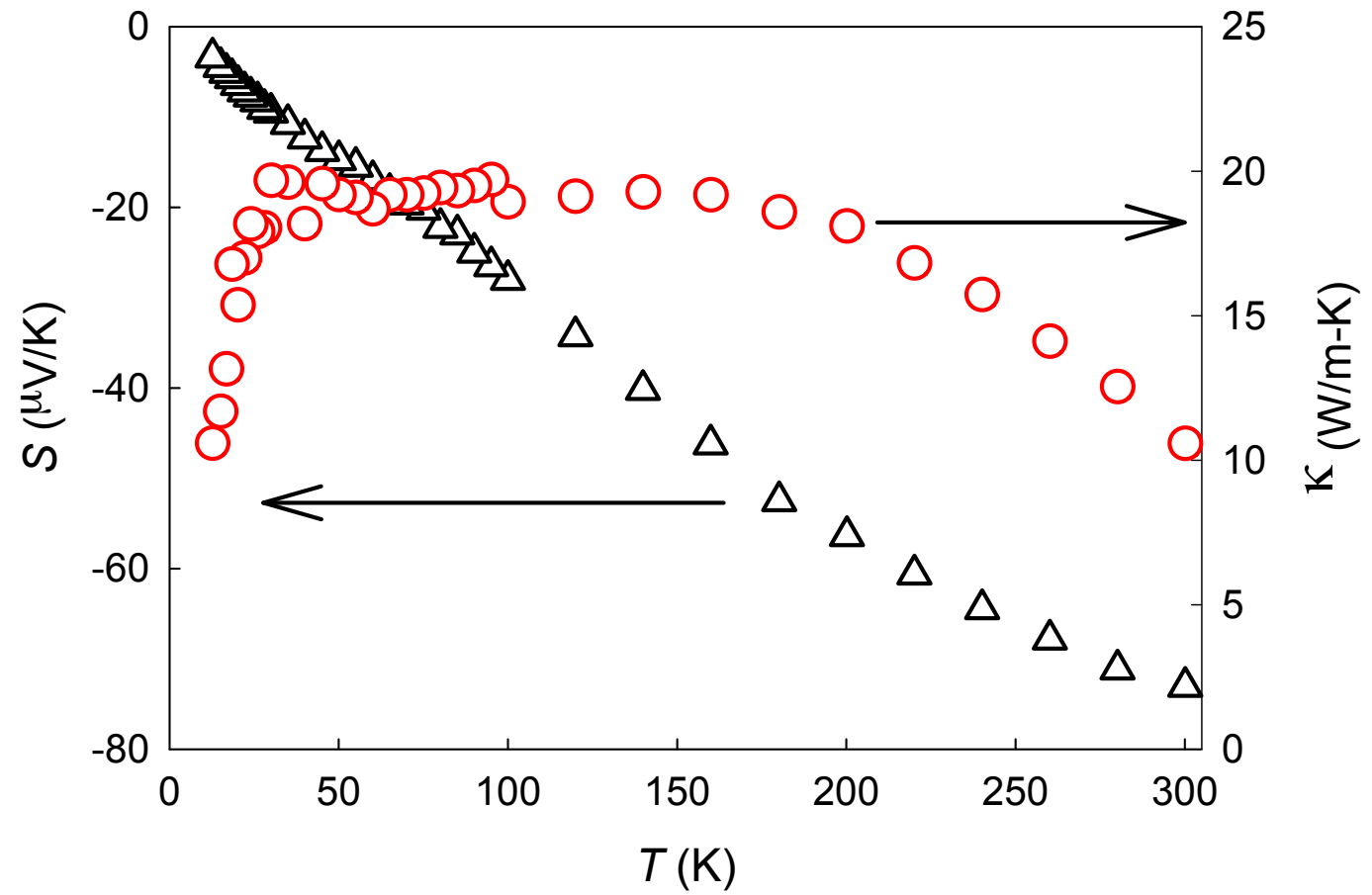

Figure 8. The Seebeck coefficient, $S$, (black triangles) and thermal conductivity, $\kappa$, (red circles) as functions of temperature for $\mathrm{Na}_{4} \mathrm{Ge}_{13}$

The absolute value of $S$ increases with temperature, as expected for heavily doped semiconductors with negligible phonon drag. The temperature dependence and magnitude of $S$ is similar to that of partially-filled, semiconducting $\mathrm{Na}_{x} \mathrm{Si}_{136}(x=2.9)$ clathrates [62]. The temperature dependence of $\kappa$ is also shown in Figure 8. The higher $\kappa$ values, as compared to those of the polycrystalline specimen [7], is a result of the crystallinity of the $\mathrm{Na}_{4} \mathrm{Ge}_{13}$ measured here. The magnitude of $\kappa$ for $\mathrm{Na}_{4} \mathrm{Ge}_{13}$ is significantly lower than that of single-crystal Ge [63], but significantly higher than more closely related clathrates [64]. The observed thermal conductivity is approximately four-times higher than that of $\left.\mathrm{Ba}_{8} \mathrm{Ge}_{43}\right]_{3}$ [65] and one order of magnitude higher than that of $\mathrm{Ba}_{6} \mathrm{Ge}_{24}$, phases which have $\mathrm{Ge}$ vacancies in addition to the disorder associated with "rattling" $\mathrm{Ba}$ ions [66]. Like $S$, the magnitude of the thermal conductivity of $\mathrm{Na}_{4} \mathrm{Ge}_{13}$ is readily comparable to that of partially-filled $\mathrm{Na}_{x} \mathrm{Si}_{136}$ clathrates [62]. The main contribution to the total thermal conductivity for $\mathrm{Na}_{4} \mathrm{Ge}_{13}$ is that of the crystal lattice (Figure S6 in the SI), with a negligible electronic thermal conductivity, consistent with the 
semiconducting behavior. Yet, the thermal conductivity seems surprisingly high compared with related systems. $\mathrm{Na}$ and $\mathrm{Ge}$ within the larger channels of the crystal structure have the largest atomic displacement parameters (Table 2) as compared to all other atomic constituents and therefore should act as effective phonon scattering centers. However, the reduced number of atoms in the unit cell and the presence of light $\mathrm{Na}$ guest atoms (compared with other Ge-based clathrate phases) will have an overall tendency to increase thermal conductivity. The underlying microscopic mechanism for the seemingly large thermal conductivity is not completely understood and is of potential interest for future investigations.

Figure 9 shows the temperature dependence of resistivity, $\rho$, for $\mathrm{Na}_{4} \mathrm{Ge}_{13}$. It decreases with increasing temperature, a confirmation of the semiconductor behavior. A fit of $\operatorname{Ln}(\rho)$ versus $1 / T$ in the highest-temperature region measured $(280-300 \mathrm{~K})$ gives a band gap value of $\sim 0.1 \mathrm{eV}$, lower than that of elemental Ge. This agrees with the DFT calculations of $0.16 \mathrm{eV}$, but should be viewed as a lower limit due to the limited temperature range. The same calculation on elemental Ge results in gap closure (see SI).

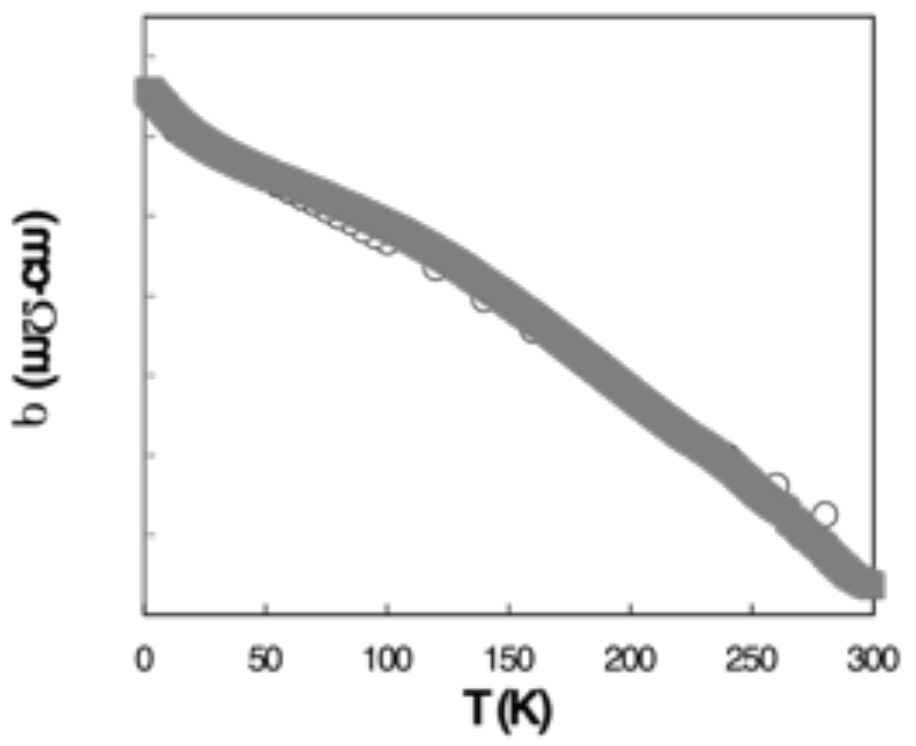

Figure 9. Electrical resistivity, $\rho$, for two independent samples of $\mathrm{Na}_{4} \mathrm{Ge}_{13}$.

\section{Conclusion}


Single-crystalline $\mathrm{Na}_{4} \mathrm{Ge}_{13}$ was synthesized for the first time by employing a high-pressure/hightemperature approach. This complex Zintl compound has a hexagonal crystal structure comprised of a three-dimensional $s p^{3} \mathrm{Ge}$ framework forming small and large channels along the crystallographic $c$-axis. The most conspicuous aspect of its intriguing crystal structure is the presence of disordered $\left[\mathrm{Ge}_{4}\right]^{4-}$ Zintl ions surrounded by six-fold $\mathrm{Na}$ rings within the larger channels, which was missing from the original $\mathrm{Na}_{1-x} \mathrm{Ge}_{3+z}$ model. This material constitutes the first example of an intermetallic system in which an exclusively Ge framework crystallizes in a channel configuration and Zintl ions are confined within these channels. The availability of specimens in the form of agglomerations of single crystals allowed for measurement of the intrinsic low-temperature transport properties of this material for the first time. Seebeck coefficient measurements indicated that $\mathrm{Na}_{4} \mathrm{Ge}_{13}$ exhibits semiconductor behavior, and this was corroborated by DFT calculations. Furthermore, it exhibits thermal conductivity that is lower than that of single-crystal $\mathrm{Ge}$, but larger than related clathrate materials. The $\mathrm{Na}_{4} \mathrm{Ge}_{13}$ composition is an example of a system with a favorable combination of electronic and thermal properties, which is a necessary condition for an effective thermal-to-electrical energy conversion, according to the phonon-glass electron-crystal concept $[5,63]$. The structureproperty characteristics of this composition suggest that additional investigation of Zintl phases is warranted in order to explore their potential for efficient thermoelectric conversion applications [67].

\section{Associated Content}

Supporting Information

Structure information, molecular orbital calculations, tetrahedra rotation analysis and electronic band structures. This material is available free of charge via the Internet at http://pubs.acs.org.

\section{Author Information}

Corresponding Author

*tstrobel@carnegiescience.edu 


\section{Notes}

The authors declare no competing financial interests.

\section{Acknowledgement}

We are grateful to Dr. R. Hoffmann for valuable input and for providing us with computational resources. We thank Dr. D.Y. Kim for useful discussions and thank Dr. Y. Fei for guidance using the multianvil press. We thank Dr. D. Popov for assistance with XRD measurements at the APS and thank Dr. S. Teat for the opportunity to use beamline 11.3.1 at the ALS. This work was supported as part of the Energy Frontier Research in Extreme Environments (EFree) Center, an Energy Frontier Research Center funded by the US Department of Energy, Office of Science under Award Number DE-SC0001057. Facilities and instrumentation support were provided by the following: Transport properties measurements and data analyses performed at USF were supported by the U.S. Department of Energy, Basic Energy Sciences, Division of Materials Science and Engineering, under Award DE-FG02-04ER46145. Single-crystal diffraction experiments performed at beamline 11.3.1 of the Advanced Light Source (ALS), Lawrence Berkeley National Laboratory, were supported by the Office of Science, Office of Basic Energy Sciences of the U.S. Department of Energy under contract No. DE-AC02-05CH11231. Work at the ALS was also partially supported by COMPRES, the consortium for Materials Properties Research in Earth Sciences under NSF Cooperative Agreement EAR 1606856. Single-crystal diffraction experiments were also performed at HPCAT (Sector 16), Advanced Photon Source (APS), Argonne National Laboratory. HPCAT operations are supported by DOE-NNSA under Award No. DE-NA0001974, with partial instrumentation funding by NSF. The Advanced Photon Source is a U.S. Department of Energy (DOE) Office of Science User Facility operated for the DOE Office of Science by Argonne National Laboratory under Contract No. DEAC02-06CH11357. Computational aspects of this work were partially supported by the Natural

Sciences and Engineering Research Council (NSERC) of Canada (RGPIN-2016-06276) and Carleton University (start-up grant 186853). 


\section{References}

1. Chemistry, Structure and Bonding of Zintl Phases and Ions, ed. Kauzlarich, S. M., VCH, New York, 1996.

2. Scharfe, S.; Kraus, F.; Stegmaier, S.; Schier, A.; Fässler, T. F. (2011), Zintl Ions, Cage Compounds, and Intermetalloid Clusters of Group 14 and Group 15 Elements. Angew. Chem. Int. Ed. 2011, 50, 3630-3670.

3. Zintl, E. Intermetallische Verbindungen. Angew. Chem. 1939, 52, 1-6.

4. Klemm, W.; Busmann, E. Volumeninkremente und Radien Einiger Einfach Negativ Gelandener Ionen. Z. Anorg. Allg. Chem. 1963, 319, 297-311.

5. The Chemistry and Physics of Inorganic Clathrates, ed. Nolas, G. S. Wiley, 2014.

6. Silicon Chemistry, ed. Jutzi P.; Schubert, U. Wiley VCH, Weinheim, 2003.

7. Beekman M.; Stefanoski S.; Wong-Ng W.; Kaduk J. A.; Huang Q., Reeg C.; Bowers C. R.; Nolas, G. S. Structure and Thermal Conductivity of $\mathrm{Na}_{1-\mathrm{x}} \mathrm{Ge}_{3+z}$. J. Solid State Chem. 2010, 183, 1272-1277.

8. Beekman M.; Kaduk J. A.; Huang Q.; Wong-Ng W.; Yang W.; Wang D.; Nolas G. S. Synthesis and Crystal Structure of $\mathrm{Na}_{1-\mathrm{x}} \mathrm{Ge}_{3+\mathrm{z}}$ : a Novel Zeolite-like Framework Phase in the $\mathrm{Na}-\mathrm{Ge}$ System. Chem. Commun. 2007, 1, 837-839.

9. Jakobsen H. J.; Bildsøe H.; Beekman M.; Stefanoski S.; Nolas G. S.; Bowers C. R. LowTemperature ${ }^{23} \mathrm{Na}$ MAS NMR Reveals Dynamic Effects and Compositions for the Large and Small Channels in the Zeolite-Like Ge-Framework of $\mathrm{Na}_{1-\mathrm{x}} \mathrm{Ge}_{3+\mathrm{z}}$ Materials. J. Phys. Chem. C 2014, 118, 28890-28897.

10. Baerlocher C.; McCusker L. B.. Database of Zeolite Structures, Available at /http://www.izastructure.org/databases/S.

11. San-Miguel, A.; Toulemonde, P. High-pressure Properties of Group IV Clathrates. High Press. Res. 2005, 25, 159-185.

12. Wosylus, A.; Veremchuk, I.; Schnelle, W.; Baitinger, M.; Schwarz, U.; Grin, Yu. Cs8-xSi46: A Type-I Clathrate with Expanded Silicon Framework. Chem. Eur. J. 2009, 15, 5901-5903. 
13. a) Yamanaka, S.; Komatsu, M.; Tanaka, M.; Sawa, H.; Inumaru, K. High-Pressure Synthesis and Structural Characterization of the Type II Clathrate Compound $\mathrm{Na}(30.5) \operatorname{Si}(136)$ Encapsulating Two Sodium Atoms in the Same Silicon Polyhedral Cages. J. Am. Chem. Soc. 2014, 136, 7717-7725. b) Yamanaka, S. Silicon Clathrates and Carbon Analogs: High Pressure Synthesis, Structure, and Superconductivity. Dalton Trans. 2010, 39, 1901-1915.

14. Kim D. Y.; Stefanoski S.; Kurakevych O. O.; Strobel T. A. Synthesis of an open-framework allotrope of silicon. Nature Mater. 2014, 14, 169-173.

15. Witte J.; von Schnering H. G. Das Verhalten der Alkalimetalle zu Halbmetallen. XI. Die Kristallstruktur von NaSi und NaGe. Z. Anorg. Allg. Chem. 1964, 327, 260-273.

16. Schäfer R.; Klemm W. Das Verhalten der Alkalimetalle zu Halbmetallen. IX. Weitere Beiträge zur Kenntnis der Silicide und Germanide der Alkalimetalle. Z. Anorg. Allg. Chem. 1961, 312, 214-220.

17. von Schnering, H. G.; Baitinger, M.; Bolle, U.; Carrillo-Cabrera, W.; Curda, J.; Grin, Y.; Heinemann, F.; Llanos, J.; Peters, K.; Schmeding, A.; Somer, M. Binary Alkali Metal Compounds with the Zintl Anions $\left[\mathrm{Ge}_{9}\right]^{4-}$ and $\left[\mathrm{Sn}_{9}\right]^{4-}$. Z. Anorg. Allg. Chem. 1997, 623, 10371039 .

18. Hoch, C.; Wendorff, M.; Röhr, C. Synthesis and Crystal Structure of the Tetrelides $A_{12} M_{17}$ ( $\mathrm{A}=\mathrm{Na}, \mathrm{K}, \mathrm{Rb}, \mathrm{Cs} ; \mathrm{M}=\mathrm{Si}, \mathrm{Ge}, \mathrm{Sn})$ and $\mathrm{A}_{4} \mathrm{~Pb} 9(\mathrm{~A}=\mathrm{K}, \mathrm{Rb})$. J. Alloys Compd. 2003, 361, $206-221$.

19. Carrillo-Cabrera, W.; Gil, R.C.; Somer, M.; Persil Ö.; von Schnering, H. G. $\mathrm{Na}_{12} \mathrm{Ge}_{17}$ : A Compound with the Zintl Anions $\left[\mathrm{Ge}_{4}\right]^{4-}$ and $\left[\mathrm{Ge}_{9}\right]^{4-}-$ Synthesis, Crystal Structure, and Raman Spectrum. Z. Anorg. Allg. Chem. 2003, 629, 601-608.

20. Cros C.; Pouchard M.; Hagenmuller P. Sur Une Nouvelle Famille de Clathrates Minéraux Isotypes des Hydrates de Gaz et de Liquides. Interprétation des Résultats Obtenus. J. Solid State Chem. 1970, 2, 570-581.

21. Dera, P.; Zhuravlev, K.; Prakapenka, V.; Rivers, M.; Finkelstein, G. J.; Grubor-Urosevic, O.; Tschauner, O.; Clark, S. M.; Downs, R. T. High-pressure Single-crystal Micro X-ray Diffraction Analysis with GSE_ADA/RSV Software. High Press. Res. 2013, 33, 466-484.

22. Spek, A. L. Structure Validation in Chemical Crystallography. Acta Cryst. 2009, D65, 148-155. 
23. Sheldrick, G. M. A Short History of SHELX. Acta Crystallogr. Sect. A: Found. Crystallogr. 2008, 6, 112-122.

24. Sheldrick, G. M. Crystal Structure Refinement with SHELXL. Acat Crystallogr. Sect. C: Struc. Chem. 2015, 71, 3-8.

25. Parthe, E.; Gelato, L. M. The Standardization of Inorganic Crystal-structure Data. Acta Cryst., $A$ 1984, 40, 169-183.

26. Gelato, L. M.; Parthe, E. STRUCTURE TIDY - a Computer Program to Standardize Crystal Structure Data. J. Appl. Cryst. 1987, 20, 139-143.

27. Palmer, D. CrystalMaker Software Version 9.2.5, CrystalMaker Software Ltd., Oxford, England, 2015.

28. Martin J.; Erickson S.; Nolas G.S.; Alboni P.; Tritt T.M.; Yang J. Structural and Transport Properties of $\mathrm{Ba}_{8} \mathrm{Ga}_{16} \mathrm{Si}_{\mathrm{x}} \mathrm{Ge}_{30-\mathrm{x}}$ Clathrates. J. Appl. Phys. 2006, 99, 044903.

29. Martin J.; Nolas G. S. Apparatus for the Measurement of Electrical Resistivity, Seebeck Coefficient, and Thermal Conductivity of Thermoelectric Materials Between $300 \mathrm{~K}$ and $12 \mathrm{~K}$. Rev. Sci. Instrum. 2016, 87, 015105.

30. Kresse, G.; Hafner, J. Ab Initio Molecular Dynamics for Liquid Metals. Phys. Rev. B 1993, $47,558-561$.

31. Kresse, G.; Hafner, J. Ab Initio Molecular-dynamics Simulation of the Liquid-metalamorphous-semiconductor Transition in Germanium. Phys. Rev. B 1994, 49, 14251-14269.

32. Kresse, G.; Furthmüller. J. Efficiency of Ab-initio Total Energy Calculations for Metals and Semiconductors Using a Plane-wave Basis Set. Comput. Mat. Sci. 1996, 6, 15-50.

33. Kresse, G.; Furthmüller. J. Efficient Iterative Schemes for ab initio Total-energy Calculations Using a Plane-wave Basis Set. Phys. Rev. B 1996, 54, 11169-11186.

34. Perdew, J. P.; Burke, K.; Ernzerhof, M. Generalized Gradient Approximation Made Simple. Phys. Rev. Lett. 1996, 77, 3865-3868.

35. Perdew, J. P.; Burke, K.; Ernzerhof, M. Generalized Gradient Approximation Made Simple [Phys. Rev. Lett. 77, 3865 (1996)]. Phys. Rev. Lett. 1997, 78, 1396.

36. Blochl, P. E. Projector Augmented-wave Method. Phys. Rev. B 1994, 50, 17953-17979. 
37. Kresse, G.; Joubert, D. From Ultrasoft Pseudopotentials to the Projector Augmented-wave Method. Phys. Rev. B 1999, 59, 1758-1775.

38. Monkhorst, H. J.; Pack, J. D. Special Points for Brillouin-zone Integrations Phys. Rev. B 1976, 13, 5188-5192.

39. Deringer, V. L.; Tchougréeff, A. L.; Dronskowski, R. Crystal Orbital Hamilton Population (COHP) Analysis As Projected from Plane-Wave Basis Sets. J. Phys. Chem. A 2011, 115, 5461-5466.

40. Maintz, S.; Deringer, V. L.; Tchougréeff, A. L.; Dronskowski, R. Analytic Projection from Plane-Wave and PAW Wavefunctions and Application to Chemical-Bonding Analysis in Solids. J. Comput. Chem. 2013, 34, 2557-2567.

41. Schmidt, M. W.; Baldridge, K. K.; Boatz, J. A.; Elbert, S. T.; Gordon, M. S.; Jensen, J. H.; Koseki, S.; Matsunaga, N.; Nguyen, K. A.; Su, S.; Windus, T. L.; Dupuis, S.; Montgomery, J. A. General Atomic and Molecular Electronic Structure System. J. Comput. Chem. 1993, 14, 1347-1363.

42. Gordon, M. S.; Schmidt, M. W. Advances in Electronic Structure Theory: GAMESS a Decade Later in Theory and Applications of Computational Chemistry: The First Forty Years. Dykstra, C. E.; Frenking, G.; Kim, K. S.; Scuseria, G. E., Eds.; Elsevier, Amsterdam, 2005, pp. 1167-1189.

43. Dunning, T. H. Gaussian Basis Sets for Use in Correlated Molecular Calculations. I. The Atoms Boron Through Neon and Hydrogen. J. Chem. Phys. 1989, 90, 1007-1023.

44. Fonari, A.; Stauffer S., https://github.com/raman-sc/VASP/, 2013.

45. Porezag, D.; Pederson, M. R. Infrared Intensities and Raman-scattering Activities Within Density-functional Theory. Phys. Rev. B, 1996, 54, 7830.

46. Kurakevych, O. O.; Strobel, T. A.; Kim, D.; Muramatsu T.; Struzhkin, V. V. Na-Si Clathrates Are High-Pressure Phases: A Melt-Based Route to Control Stoichiometry and Properties. Cryst. Growth Des. 2013, 13, 303-307.

47. Carrillo Cabrerra, W.; Borrmann, H.; Paschen, S.; Baenitz, M.; Steglich, F.; Grin, Y. Ba $6 \mathrm{Ge}_{25}$ : Low-temperature Ge-Ge Bond Breaking During Temperature-induced Structure Transformation. J. Solid State Chem. 2005, 178, 715-728. 
48. Stegmaier, S.; Waibel, M.; Henze, A.; Jantke, L.A.; Karttunen, A. J.; Fassler, T. F. Soluble Zintl Phases $\mathrm{A}_{14} \mathrm{ZnGe}_{16}(\mathrm{~A}=\mathrm{K}, \mathrm{Rb})$ Featuring $\left[\left(\eta^{3}-\mathrm{Ge}_{4}\right) \mathrm{Zn}\left(\eta^{2}-\mathrm{Ge}_{4}\right)\right]^{6-}$ and $\left[\mathrm{Ge}_{4}\right]^{4-}$ Clusters and the Isolation of $\left[(\mathrm{MesCu})_{2}\left(\eta^{3}, \eta^{3}-\mathrm{Ge}_{4}\right)\right]^{4-}$ : The Missing Link in the Solution Chemistry of Tetrahedral Group 14 Element Zintl Clusters. J. Am. Chem. Soc. 2012, 134, 14450-14460.

49. Nolas, G. S.; Kendziora, C. A.; Gryko, J.; Dong, J.; Myles, C. W. Raman Scattering Study of Stoichiometric Si and Ge type II Clathrates. J. Appl. Phys. 2002, 92, 7225-7230.

50. Shimizu, H.; Iitaka, T.; Fukushima, T.; Kume, T.; Sasaki, S.; Sata, N.; Ohishi, Y.; Fukuoka, H.; Yamanaka, S. Raman and X-ray Diffraction Studies of Ba doped Germanium Clathrate $\mathrm{Ba}_{8} \mathrm{Ge}_{43}$ at High Pressures. J. Appl. Phys. 2007, 101, 063549.

51. Shimizu, H.; Fukushima, T.; Kume, T.; Sasaki, Y.; Fukuoka, H.; Yamanaka, S. High-pressure Raman Study of Ba doped type-III Germanium Clathrate $\mathrm{Ba}_{24} \mathrm{Ge}_{100}$ up to $26 \mathrm{GPa}$. J. Appl. Phys. 2007, 101, 113531.

52. Ormeci A.; Aydemir, U.; Somer. M. Vibrational Spectra and Quantum Chemical Calculations of the Pure and Mixed Cluster Anions $\left[\mathrm{Si}_{\mathrm{x}} \mathrm{Ge}_{4-\mathrm{x}}\right]^{4-}$ and $\left[\mathrm{Ge}_{\mathrm{x}} \mathrm{Sn}_{4-\mathrm{x}}\right]^{4-}(\mathrm{x}=0-4)$ in Compounds with Potassium and Cesium. Z. Anorg. Allg. Chem. 2011, 637, 907-914.

53. Somer M.; Aydemir, U.; Baitinger, M.; von Schnering, H. G. Vibrational Spectra of Cluster Anions. 2. Vibrational Spectra of Compounds with the Cluster Anions $\left[\mathrm{E}_{4}\right]^{4-}: \mathrm{M}_{4} \mathrm{E}_{4}(\mathrm{M}=\mathrm{K}, \mathrm{Rb}$, $\mathrm{Cs} ; \mathrm{E}=\mathrm{Ge}, \mathrm{Sn})$ and $\beta-\mathrm{Na}_{4} \mathrm{Sn}_{4}$. Z. Anorg. Allg. Chem. 2006, 632, 1281-1286.

54. Carrillo-Cabrera, W.; Gil, R. C.; Somer, M.; Persil, O.; von Schnering, H. G. Z. $\mathrm{Na}_{12} \mathrm{Ge}_{17}$ : A Compound with the Zintl Anions $\left[\mathrm{Ge}_{4}\right]^{4-}$ and $\left[\mathrm{Ge}_{9}\right]^{4-}$ - Synthesis, Crystal Structure, and Raman Spectrum. Anorg. Allg. Chem. 2003, 629, 601-608.

55. Dronskowski, R.; Bloechl, P. E. Crystal orbital Hamilton populations (COHP): energyresolved visualization of chemical bonding in solids based on density-functional calculations. $J$. Phys. Chem. 1993, 97, 8617-8624.

56. Zurek, E.; Yao, Y. Theoretical Predictions of Novel Superconducting Phases of BaGe $\mathrm{Stable}$ at Atmospheric and High Pressures. Inorg. Chem. 2015, 54, 2875-2884.

57. Atoms in Molecules: A Quantum Theory, Bader, R. F. W. Oxford University Press, 1994.

58. Sanville, E.; Kenny, S. D.; Smith, S.; Henkelman, G. Improved grid-based algorithm for Bader charge allocation. J. Comput. Chem. 2007, 28, 899-908. 
59. Stefanoski S.; Malliakas, C. D.; Kanatzidis, M. G.; Nolas, G. S. Synthesis and Structural Characterization of $\mathrm{Na}_{\mathrm{x}} \mathrm{Si}_{136}(0<\mathrm{x} \leq 24)$ Single Crystals and Low-Temperature Transport of Polycrystalline Specimens. Inorg. Chem. 2012, 51, 8686-8692.

60. Stefanoski S.; Martin J.; Nolas G. S. Low Temperature Transport Properties and Heat Capacity of Single-crystal Nasisi46. J. Phys.: Condens. Matter. 2010, 22, 485404.

61. Beekman M.; Baitinger M.; Borrmann H.; Schnelle W.; Meier K.; Nolas G. S.; Grin Y. Preparation and Crystal Growth of $\mathrm{Na}_{24} \mathrm{Si}_{136}$. J. Am. Chem. Soc. 2009, 131, 9642-9643.

62. Beekman M.; Schnelle W.; Borrmann H.; Baitinger M.; Grin Yu.; Nolas G. S. Intrinsic Electrical and Thermal Properties from Single Crystals of $\mathrm{Na}_{24} \mathrm{Si}_{136}$. Phys. Rev. Lett. 2010, 104, 018301.

63. Glassbrenner C. J.; Slack G. A. Thermal Conductivity of Silicon and Germanium from $3^{\circ} \mathrm{K}$ to the Melting Point. Phys. Rev. 1964, 13, A1058-A1069.

64. The Physics and Chemistry of Inorganic Clathrates, Nolas, G. S. (Ed). Springer, 2014.

65. Aydemir, U.; Candolfi, C.; Borrmann, H.; Baitinger, M.; Ormeci, A.; Carrillo-Cabrera, W.; Chubilleau, C.; Lenoir, B.; Dauscher, A.; Oeschler, N.; Steglich, F.; Grin, Yu. Crystal structure and transport properties of $\mathrm{Ba}_{8} \mathrm{Ge}_{43}$ ?. Dalton Trans. 2010, 39, 1078-1088.

66. Kim, S. J.; Hu, S.; Uher, C.; Hogan, T.; Huang, B.; Corbett, J. D.; Kanatzidis. M. G. Structure and Thermoelectric Properties of $\mathrm{Ba}_{6} \mathrm{Ge}_{25}-\mathrm{x}, \mathrm{Ba}_{6} \mathrm{Ge}_{23} \mathrm{Sn}_{2}$, and $\mathrm{Ba}_{6} \mathrm{Ge}_{22} \mathrm{In}_{3}$ : Zintl Phases with a Chiral Clathrate Structure. J. Solid State Chem. 2000, 153, 321-329.

67. Kauzlarich, S. M.; Brown, R. S.; Snyder, G. J. Zintl Phases for Thermoelectric Devices. Dalton. Trans. 2007, 2099-2107. 

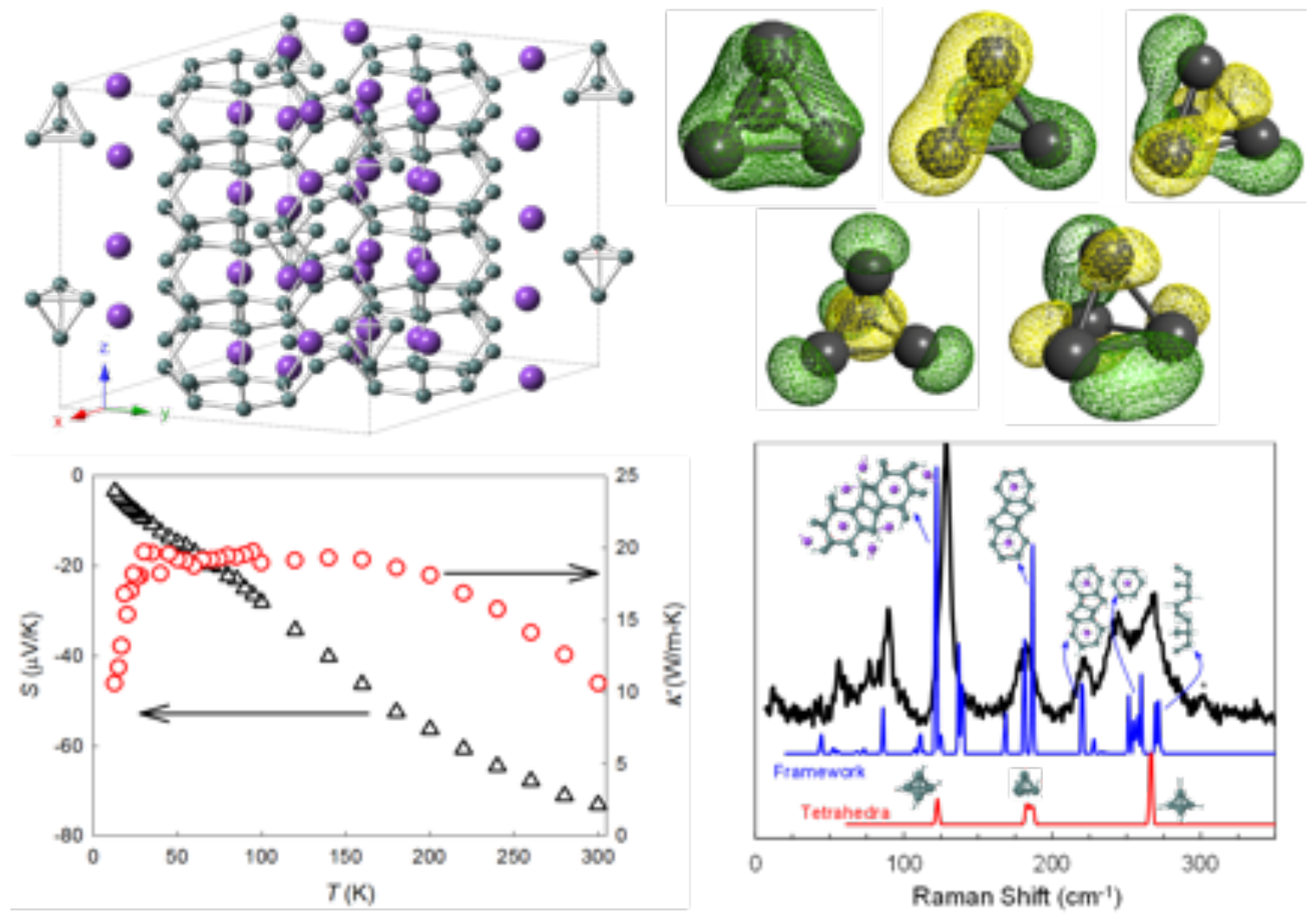

\section{Synopsis:}

$\mathrm{Na}_{4} \mathrm{Ge}_{13}$ is a framework structure with small and large channels that hold $\mathrm{Na}$ and is the first known example of a guest-host structure where discrete $\left(\mathrm{Ge}_{4}\right)^{4-}$ Zintl polyanions are confined inside the channels of a three-dimensional covalent framework. These features give rise to temperature-dependent disorder, as confirmed by first principles calculations and physical properties measurements. 